\title{
An efficient methodology for quantification of synergy and antagonism in Single Electron Transfer antioxidant assays
}

\author{
Prieto M.A. ${ }^{* 1}$, Curran, Thomas P. ${ }^{2}$, Gowen, A. ${ }^{2}$ \& Vázquez J.A. ${ }^{1}$ \\ ${ }^{1}$ Grupo de Reciclado y Valorización de Materiales Residuales (REVAL), Instituto de Investigacións \\ Mariñas (IIM-CSIC), Vigo, Spain.
}

${ }^{2}$ UCD School of Biosystems Engineering, University College Dublin, Belfield, Dublin 4, Ireland.

*Corresponding author E-mail: michaelumangelum@gmail.com

\begin{abstract}
The development of new antioxidant compounds for incorporation in foods is a rapidly growing research area. The resulting interactions between complex antioxidant mixtures is a key issue, however, research in this area is still in its infancy. Experimental antioxidant models based on conventional dose-responses, that can predict joint effects of chemical mixtures, are urgently needed. This paper illustrates a methodological procedure for Single Electron Transfer (SET) antioxidant assays to determine the synergistic and antagonistic effects of combining binary mixtures of antioxidants. Despite the abundance of theories and procedures to describe the synergistic/antagonistic effects in SET assays, they appear to be inadequate. Some features hindering advances in this field include the lack of: (1) experimental design, as a result of the extended use of unambiguous and simplistic procedures to quantify the effects of joint responses, based on singledose values; (2) detailed mathematical hypotheses to quantify dose-response values, which in addition causes the associated difficulties for assessing the statistical consistence of the results; and (3) functional approaches that consider the possibility of interactive effects. This paper proposes solutions for each of these limitations. Established ideas from existing fields are used to replace the current simplistic procedures, in order to quantify the effects of joint responses. One of the common hypothesis (known as concentration addition) for describing the combined effects is established for SET assays. A dose dependent mathematical model representative of this hypothesis, based on probability functions with meaningful parameters, is applied. The interactive effects between antioxidants are introduced into the model with simple auxiliary functions that describe the variations induced by each antioxidant in the parameters that define the effects of the other. Finally, a comprehensive index to summarize the complex parametric responses in one single value is proposed. Although the approach was experimentally demonstrated just in two classical SET assays (DPPH and ABTS), the results could be directly expanded in future to other types of classical SET assays. The methodology proposed is more complex than some relatively common approaches; nevertheless we believe that it is free of the controversial aspects listed above. Statistically consistent responses of null, synergy and antagonism effects were found when characterizing the interactions between several pairs of individual and complex mixtures of chemical antioxidant agents.
\end{abstract}

\section{Keywords:}

dose-response analysis; synergy and antagonism; mechanisms of interaction; antioxidant interaction; SET assay

\section{Chemical compounds studied in this article:}


Butyl-hydroxyanisole (CID 24667); propyl 3,4,5-trihydroxybenzoate (CID 4947); butylhydroxytoluene (CID 15570435); 6-ethoxy-2,2,4-trimethyl-1,2-dihydroquinoline (CID 3293); 6hydroxy-2,5,7,8-tetramethylchroman-2-carboxylic acid (CID 40634); (2R)-2,5,7,8-tetramethyl-2[(4R,8R)-(4,8,12-trimethyltridecyl)]-6-chromanol (CID 14985); and (5R)-[(1S)-1,2-dihydroxyethyl]3,4-dihydroxyfuran-2(5H)-one (CID 54670067).

\section{INTRODUCTION}

The antioxidant assays that evaluate the direct effectiveness of compounds against free radical species can be divided into two main reaction categories, depending on the mechanism involved, some assumptions and assessment type (Apak et al. 2013): 1) Hydrogen atoms transfer (HAT) which measures the classical ability of an antioxidant to quench free radicals by hydrogen donation (Prieto et al. 2012); and 2) Single electron transfer (SET) which detects the ability of a potential antioxidant to transfer one electron to reduce any compound, including metals, carbonyls, and radicals (Huang et al. 2005).

The authors consider that HAT-based methods are most relevant to reactions where antioxidants typically act. HAT-based reactions are solvent and $\mathrm{pH}$ independent, but the presence of reducing agents, including metals, can lead to erroneously high apparent reactivity (Roginsky and Lissi 2005). SET-based reactions are $\mathrm{pH}$ dependent, and the correlation between the different SET methods is significant (Niki 2010) but not consistent due to the interference of trace components and contaminants (particularly metals), which causes variability and poor reproducibility. When monitoring the reaction pathway, it is very difficult to distinguish between HAT and SET reactions, and the two reactions may take place simultaneously. There is certain agreement that complete HATbased assays include ORAC (Oxygen Radical Absorbance Capacity), TRAP (Total Radical-Trapping Antioxidant Parameter), CBA (Crocin Bleaching Assay), $\beta$ BA ( $\beta$-carotene Bleaching Assay) and OxHIIA (Oxidative Hemolysis Inhibition Assay). SET-based assays include ABTS, DPPH, FCR (Folin-Ciocalteu reagent), FRAP, ferricyanide and CUPRAC.

In the food technology, pharmaceutical and medical fields the interest for understanding the effects of multiple chemicals is overwhelming (Sanchez-Moreno 2002). It is impossible to test every chemical combination. However, it is desirable to be able to predict effects of mixtures based on knowledge of the effects of simple binary combinations. From previous research (Bruun-Jensena and Skovgaardb 1994; Peyrat-Maillard, Cuvelier, and Berset 2003; Yang et al. 2009), when more than one antioxidant is present in a controlled chemical environment (HAT or SET), the final antioxidant capacity (AC) found is higher or lower than expected in many cases. However, in these fields of study, the concepts of synergy and antagonism are often characterized by simplistic relationships between the individual effects and very basic experimental procedures (Jia et al. 1998; Marinova, Toneva, and Yanishlieva 2008; Yang et al. 2009), rather than generalizing the classical approaches from the risk assessment in the toxicological area (Berenbaum 1985a, 1985b; Bliss 1937, 1939; Greco, Bravo, and Parsons 1995; Loewe and Muischnek 1926). In addition, graphical approaches that avoid the application of response surface models are poorly suited to discern the joint effect of binary mixtures of antioxidants. This paper pursues a solution for each of these limitations.

Previously, mathematical tools have been developed to provide an algebraic environment to translate the classical interactive hypothesis regarding the binary combination of individual chemical entities (Murado and Prieto 2013a). Furthermore, a procedure has been described to identify and quantify the interactive effects between two antioxidants in HAT assays (Prieto, Murado, and Vázquez 2014b), and applied to investigate interactive mechanisms in complex mixtures of antioxidants (Prieto and Vázquez 2014). In this study, we continue with the application of theoretical 
standpoints to different fields, by cautiously describing a method for SET antioxidant assays to determine the synergistic and antagonistic effects of combining binary mixtures of antioxidants. We have transferred the more recent advances in the quantification of the dose effect of individual agents and the joint effect of binary mixtures through response surface analysis. The procedure was tested in two well-known SET assays, the DPPH and ABTS methods. They were selected because they provide an optimized response system that is fairly representative of the SET oxidation processes, especially accurate, reproducible and yields a low experimental error (Floegel et al. 2011). They are extensively used to quantify the potential AC (antioxidant capacity). The respective protocols have been repeatedly revised and improved, being well optimized at present (Nabavi et al. 2013).

Despite the effort of researchers (Jia et al. 1998; Marinova, Toneva, and Yanishlieva 2008; Yang et al. 2009) to describe the synergistic/antagonistic effects in SET assays, the lack of theoretical standpoints provided by classical approaches from other field of studies, may have prevented researchers to find more conclusive solutions. This paper illustrates a methodological procedure for SET antioxidant assays to determine the synergistic and antagonistic effects of combining binary mixtures of antioxidants.

\section{MATERIAL AND METHODS}

\subsection{Two classical Single Electron Transfer methods to assess antioxidants}

\subsubsection{DPPH bleaching reagent}

The $\mathrm{DPPH}^{\bullet+}$ radical scavenging activities were assessed as described previously (Jiménez-Escrig et al. 2000; Sharma and Bhat 2009). Briefly, 1,1-Diphenyl-2-picryl-hydrazyl (DPPH) stock solution was prepared in methanol $(0.50 \mathrm{~g} / \mathrm{L})$. For testing, the potential of antioxidants the stock solution was diluted ten times to provide an absorbance of $\sim 1.2$ units at $515 \mathrm{~nm}$.

\subsubsection{ABTS bleaching reagent}

The $\mathrm{ABTS}^{\circ+}$ radical scavenging activities were assessed as described previously (Obón et al. 2005; Re et al. 1999). Briefly, the stock solution was prepared in distilled water water by mixing $4 \mathrm{mM}$ $(21.95 \mathrm{mg} / 10 \mathrm{~mL})$ of ABTS (2,2' - azinobis-(3-ethyl-benzothiazoline- 6-sulphonic acid)) with 1.5 $\mathrm{mM}$ of potassium persulfate $\left(\mathrm{K}_{2} \mathrm{~S}_{2} \mathrm{O}_{8}, 4.03 \mathrm{mg} / 10 \mathrm{~mL}\right)$. In order to convert ABTS completely into its radical cation $\left(\mathrm{ABTS}^{\circ+}\right)$, the reaction mixture was left in the dark at room temperature for $12-16 \mathrm{~h}$, before its use. For testing the potential of antioxidants, the stock solution was diluted 22 times with phosphate buffered saline (5 mM, pH 7.4) to provide an absorbance $\sim 1.2$ units at $414 \mathrm{~nm}$.

Both stock solutions were kept in a translucent tube at $4{ }^{\circ} \mathrm{C}$ in darkness. The solvents and other chemicals used for the preparation of both reagents were of analytical grade. The absorbance of both stock radical solutions was measured daily. If any loss of the free radical activity was noticed, the solution was prepared again (Ozgen et al. 2006)

\subsubsection{Procedure for the determination of the individual dose-response effects}

The procedure was performed by adding $50 \mu \mathrm{L}$ of sample and $250 \mu \mathrm{L}$ of reagent into the wells $(350$ $\mu \mathrm{L}$ ) of a microplate reader of 96 units (Thermo Scientific Nunc 96-Well Polypropylene MicroWell Plate with flat bottom). The microplate reader (Multiskan Spectrum Microplate Photometers from Thermo Fisher Scientific) was programmed to read the absorbance at the respective wave lengths every minute during a period of 200 minutes with agitation at 660 cycles $/ \mathrm{min}$ ( $1 \mathrm{~mm}$ amplitude). The 
reduction of $\mathrm{ABTS}^{\circ+}$ and $\mathrm{DPPH}^{\circ+}\left(15 \mathrm{nM}\right.$ and $30 \mathrm{nM}$, respectively in the final solution) at $30^{\circ} \mathrm{C}$ were followed by monitoring the decrease in absorbance until the reaction reached a steady state (Serpen et al. 2007).

\subsubsection{Procedure for the determination of the synergistic and antagonistic effects between two pairs of antioxidants}

As described in related works (Prieto et al. 2014b; Prieto and Vázquez 2014), microplate assays were carried out based on a complete design for $8 \times 8$ arrays of two antioxidant mixtures at equally increasing concentrations (64 independent dose combinations) which were freshly prepared. Thus, $25 \mu \mathrm{L}$ of each antioxidant solution was added to each well containing $250 \mu \mathrm{L}$ of the corresponding reagent. All other conditions were the same as previously described.

\subsection{Single value to assess the response of antioxidants}

SET assays are rapid assays in which the indicator and radical species of the reaction can be adjusted to extend or shorten the time of the reaction. SET-based assays generally set a fixed time to measure color change of the radical. The initially chosen fixed points have a relevant impact on the final results obtained (Huang et al. 2005; Prieto et al. 2014a). Therefore, in SET assays, to avoid generating inconsistent responses, an intermediate approach must be found. Otherwise, from a mathematical point of view, we are forced to reject any result (Prieto et al. 2014b; Arts 2004). As for all non-linear kinetic reactions, the only useful rate value would be the maximum one. But, the time at which the rate reaches its maximum cannot be established a priori and varies for each compound and concentration. Therefore, for SET assays, the only possible reproducible value to be used is the final end point, which accounts for the entire potential capacity, but neglects the kinetic aspect of the reaction. One drawback of neglecting the kinetic analysis is that two antioxidants could have the same dose-response potential capacity but very different affinities towards the radical. Because the time to achieve such responses is not taken into account, their general $\mathrm{AC}$ would be registered as identical, but they are not.

Before any value to summarize the responses is considered, the responses must first be properly standardized. The best solution could be to rearrange the response as a function of the reduced radical molecules, as follows:

$$
R(t, d)=R_{0}^{\bullet+} \times\left(1-\frac{A_{t}^{d}}{C_{t}}\right)
$$

in which $R$ is the bleached radical response measured as a function of time $(t)$ and dose $(d)$ of an antioxidant. $R_{0}^{\bullet^{+}}$is the initial concentration of the radicalized form of the compound. $A$ and $C$ are the kinetic curves for each dose of the antioxidant and the control, respectively. By carrying out this standardization, the response is valid for the ABTS and DPPH responses. For other SET assays the rearrangements may need to be modified, but the essence should be kept.

For the determination of the asymptotic end point values of the kinetic response, several possible solutions exist. One of them is the kinetic analysis with explicit mathematical equations. In this sense, we have found three groups of alternatives in the literature (Murado and Vázquez 2010; Özilgen and Özilgen 1990; Terpinc, Bezjak, and Abramovič 2009), covering a wide spectrum of profile responses, from potential to sigmoid ones, with and without intercepts. These mathematical 
tools facilitates the accurate prediction of the asymptotic end point value of the kinetic part of the response for all the doses assessed. However, this analysis is rarely applied in microplate readers for SET assays, because for some antioxidants the affinity towards the radical is rapid (kinetic completion in less than $1 \mathrm{~min}$, such as trolox), while for others it is very slow (kinetic completion during more than $60 \mathrm{~min}$, such as BHT). In this sense, when testing a set of combined dose-effects (useful for evaluating the synergistic or antagonistic effects of a mixture of compounds) the resulting experiment becomes unfeasible. Therefore, other more intuitive solutions must be applied. A valuable alternative is based on the kinetic variation with the control. However, if the kinetic perspective of the response is not clear, as occurs frequently in most SET assays, we should still follow the reaction kinetics experimentally and select those values as the asymptotic ones that would not show any changes with regard to the reduction of the control.

\subsection{Illustrative set of samples: individual chemical entities and complex mixture of antioxidants}

\subsubsection{Individual chemical entities: commercial antioxidants}

(a) Butyl-hydroxyanisole (BHA): a synthetic food additive (E320) mainly used as an antioxidant and preservative. Its known capacity is suitable in lipophilic and hydrophilic environments.

(b) Butyl-hydroxytoluene (BHT): a synthetic lipophilic (fat-soluble) organic compound, chemically a derivative of phenol that is useful for its antioxidant properties. It is primarily used as a food additive (E321).

(c) Propyl 3,4,5-trihydroxybenzoate or propyl gallate (PG): an antioxidant that has been added to foods containing oils and fats to prevent oxidation (E310).

(d) (2R)-2,5,7,8-tetramethyl-2-[(4R,8R)-(4,8,12-trimethyltridecyl)]-6-chromanol or $\alpha$-tocopherol (TOC): a natural fat-soluble organic compound (E306) consisting of various methylated phenols (a type of tocopherol or vitamin E), that is useful for its antioxidant properties.

(e) 6-ethoxy-2,2,4-trimethyl-1,2-dihydroquinoline or ethoxyquin (ETX): commonly used as a food preservative (E324) in pet foods to prevent the rancidification of fats, in spices to prevent color loss due to oxidation of the natural carotenoid pigments and as a pesticide.

(f) $\quad L$-hexuronic Acid (vitamin C) or Ascorbic Acid (AA): a naturally occurring hydrosoluble organic compound with antioxidant properties. Ascorbic acid and its sodium, potassium, and calcium salts are commonly used as antioxidant food additives (E300-304).

(g) Tert-Butylhydroquinone (TBHQ): It is a derivative of hydroquinone, substituted with tertbutyl group. TBHQ is a highly effective antioxidant in foods (E319). It is added to a wide range of foods, with the highest limit $(1000 \mathrm{mg} / \mathrm{kg})$ permitted for frozen fish and fish products.

(h) 6-hydroxy-2,5,7,8-tetramethylchroman-2-carboxylic acid (Trolox, TRO): A water-soluble analog of vitamin $\mathrm{E}$ used in biological or biochemical applications to reduce oxidative stress or damage.

All compounds were purchased from Sigma S.A. (St. Louis, MO, USA). The purity of all compounds tested was higher than $98 \%$.

\subsubsection{Complex mixture of antioxidants: natural extracts from classical beverages with high content of antioxidant compounds}

Tea and coffee are aromatic beverages (Perva-Uzunalić, Škerget, Knez, Weinreich, Otto, \& Grüner, 2006), second and third most consumed after water. Their water extracted compounds present different types of antioxidants of different potential degree (Chan, Lim, Chong, Tan, \& Wong, 2010); therefore, it was considered as an excellent food case study. It has been argued that the consumption of those beverages is beneficial for health; among others, for its antioxidant activity, 
mainly due to the presence of natural antioxidants such as vitamins (mainly A, B6, C and E), polyphenols (xavonoids, xavanols, xavonols, isoxavones, quercetin, catechin, epicatechin, etc.), coenzyme Q10, carotenoids, selenium, zinc and phytochemicals (Abdullin, Turova, \& Budnikov, 2001; Sakanaka, Tachibana, \& Okada, 2005; Vinson \& Dabbagh, 1998).

Unroasted coffee beans and loose unblended tea samples, free of additives (especially the antioxidant ones), were collected, cleaned, vacuum-packed and sent to the laboratory. Coffea arabica beans (C) were harvested in 2013 in Australia. Green (China Sencha) unblended tea (T) was harvested in China in 2011. The coffee bean and unblended tea samples were weighed, grounded, sieved with a mesh size $(<0.5 \mu \mathrm{m})$ and packed in low density polyethylene pouches and preserved at $4-6{ }^{\circ} \mathrm{C}$ for further analysis. Four consecutive autoclave extractions with $100 \mathrm{~mL}$ of distilled water at $105^{\circ} \mathrm{C}$ for $60 \mathrm{~min}$ were applied to $10 \mathrm{~g}$ of each sample. The extracted material was centrifuged several times and the supernatant was filtered through Whatman glass microfibre filters (GF/D and GF/F), lyophilized and preserved at $-20{ }^{\circ} \mathrm{C}$ (Almajano et al. 2007; Perva-Uzunalić et al. 2006). All extractions were performed in triplicate and analytical methods for determination of $\mathrm{AC}$ were performed in the following days after the extraction.

\subsubsection{Antioxidant concentration ranges}

The concentration ranges used for each antioxidant sample are presented as weight used for the final reaction volume of a microplate well $(300 \mu \mathrm{L})$. Thus, for the DPPH reaction the concentrations ranges are: TRO $(0.00$ to $3.15 \mu \mathrm{g})$; $\mathrm{AA}(0.00$ to $3.15 \mu \mathrm{g})$; $\mathrm{PG}(0.00$ to $1.25 \mu \mathrm{g})$; BHA $(0.00$ to 5.00 $\mu \mathrm{g})$; BHT $(0.00$ to $17.50 \mu \mathrm{g})$; TBHQ $(0.00$ to $2.00 \mu \mathrm{g})$; ETX (0.00 to $3.15 \mu \mathrm{g})$; TOC (0.00 to 3.15 $\mu \mathrm{g})$; $\mathrm{C}(0.00$ to $6.25 \mu \mathrm{g})$; and $\mathrm{T}(0.00$ to $2.50 \mu \mathrm{g})$. While the concentration ranges in $\mu \mathrm{M}$ of the antioxidants used for the ABTS reaction are: TRO (0.00 to $0.75 \mu \mathrm{g})$; AA (0.00 to $2.00 \mu \mathrm{g})$; PG $(0.00$ to $0.25 \mu \mathrm{g})$; BHA $(0.00$ to $0.40 \mu \mathrm{g})$; BHT $(0.00$ to $2.00 \mu \mathrm{g})$; TBHQ $(0.00$ to $0.40 \mu \mathrm{g})$; ETX (0.00 to $0.75 \mu \mathrm{g})$; TOC (0.00 to $2.00 \mu \mathrm{g}) ; \mathrm{C}(0.00$ to $2.00 \mu \mathrm{g})$; and $\mathrm{T}(0.00$ to $0.75 \mu \mathrm{g})$.

\subsection{Numerical methods}

Simulated and experimental results were adjusted to the proposed models by non-linear least squares methods (quasi-Newton), using Solver complement in Excel. Parametric estimations were performed by incorporating the 'SolverAid' macro (Prikler 2009) for estimating the confidence intervals. Model consistency student's $t$ and Fisher's $F$ tests, with $\alpha=0.05$ in both cases were used. An automatic stepwise regression method was programmed in Microsoft Excel 2003 for the analysis of responses in order to test all possible parameter combinations. The following steps were applied routinely: 1) fitting the parameters from the individual responses (without interactions), using Eq. (6) for the $C A$ hypothesis; 2) using the estimates as the starting values for assaying all possible parameter combinations of Eq. (8) ( $C A, 13$ parameters and 8.191 combinations); 3) rejecting those options that lead at least to a non-statistically significant coefficient; and 4) selecting the most significant solutions, which are automatically ranked with several model selection criteria (Prieto et al. 2014b).

\section{RESULTS \& DISCUSSION}

This study investigates theoretical standpoints from different fields to develop a methodological procedure for SET antioxidant assays in order to determine the synergistic and antagonistic effects of combining two antioxidants. The classical interactive hypothesis of CA and IA for two agents was transferred by current analytical tools (Murado and Prieto 2013a). The structure of the methodological procedure is based on similar ones recently published (Prieto et al. 2014b; Prieto and Vázquez 2014) to identify and quantify the interactive effects in HAT. However, before highlighting 
the core of the problem, other issues need to be addressed such as: 1) which mathematical model is most appropriate to compute and compare the individual dose-effects of antioxidants in SET assays; 2) which of the classical interactive hypotheses (CA and IA), if any, adapts better to the chemical conditions in SET assays; 3) which descriptive surface models are currently available and what are the possible ways to summarize the complex responses scenarios in a comprehensive index; 4) what range of concentrations is more suitable for the determination of synergistic and antagonistic effects; 5) what is the maximum number of antioxidants that can be tested for their combined effect; and 6) from a theoretical standpoint, which compounds, individual antioxidants or complex mixtures, such as natural extracts, can be tested? .

Once these points are discussed, an illustrative step by step example of the methodological procedure is depicted. Finally, two classical SET assays, based on the bleaching of the $\mathrm{DPPH}^{\circ+}$ and $\mathrm{ABTS}^{\circ+}$ radicals, are used to experimentally test the procedure between the binary mixtures of the antioxidants described in the material and methods section, covering the analysis of individual chemical entities and complex mixtures of natural extracts.

\subsection{Tying up the loose ends for analyzing the response in SET assays}

\subsubsection{Dose-response model to describe the variations of the asymptotic potential values of SET assays}

HAT-based assays measure the reactivity or capacity of antioxidants on competition kinetic grounds, in which the indicators of the reaction (in general another antioxidant) compete for the reactive species with the antioxidant sample to be tested. Therefore, they rank the affinity of antioxidants to counteract the reactive species against the indicator. Because both the dose of the antioxidant and the kinetics of the process are relevant, researchers have started to use time-dose dependent models to evaluate their responses. SET-based assays measure the potential capacity of an antioxidant to counteract an oxidant that changes its color when reduced and avoids the analysis of the antioxidant kinetic affinity towards the radical species. The degree of color change (either an increase or decrease) is correlated to the concentration of antioxidants in the sample. For SET assays, when neglecting the kinetic aspect of the response, the only possible reproducible value to be used is the final one, which accounts for the entire potential capacity, but this approach ignores the affinity of the antioxidant for the radical compound.

Commonly, the linear range of the dose-response of an established commercial antioxidant (such as trolox or BHT) is used as a calibration curve to compute the equivalent potential AC of new samples, and the samples are only tested at one single dose. This simplification causes deficiencies and the risk of inconclusive results (Frankel and Finley 2008). Due to the inconsistency of the results, researchers frequently select the "linear range" of the dose-response of all samples and evaluate their AC by contrasting their respective slopes. Less commonly, authors have suggested that some radicalgenerating property of the system can be saturated (Arts 2004; Gieseg and Esterbauer 1994), and in general, the non-linearity of the dose-responses of compounds has been described by mathematical expressions common to many fields of study to evaluate the dose-responses.

The preferable options are always models that have a lower number of parameters and models with parameters that provide direct meaning of the processes under analysis. Among the most common, hyperbolic, potential or sigmoid functions are traditionally used in biological systems due to their manageability. The most appropriate models that maximize possible responses and minimize the number of parameters are sigmoid functions. In general, the three parameter sigmoid group of functions (such as the Logistic, Weibull, Hill, Gompertz or Richards-Chapman) is the best solution 
to fit individually the asymptotic endpoint values corresponding to a series of increasing levels of an antioxidant. After testing those models, it was found that the Weibull survival distribution function (Weibull and Sweden 1951) was the most satisfactory with the highest level of accuracy. The Weibull model was previously reported to successfully describe the response in other antioxidant methods (Prieto et al. 2012, 2014a). Thus, the dose-response of the asymptotic end point values can be fitted to the following equation rearranged for the purposes of this study:

$$
R(A)=K \exp \left[-\ln 2(A / \tau)^{\alpha}\right] \quad \text { briefly; } \quad R(A)=W(A ; K, m, \alpha)
$$

in which the parameter $K$ is the maximum radical reduced, asymptotic value of the response (for the SET assays studied here, reduced molecules of the radical). The parameter $\tau$ corresponds to the dose required for $50 \%$ radical reduction (substrate half-dose), which is the value of maximal predictability, because it corresponds also to the average radical molecules reduced per molecule of $A$ agent. The $\alpha$ shape parameter is related to the maximum slope of the response. Eq. (2) is very versatile: when $\alpha<1$, it can adjust the profiles of potential responses; when $\alpha=1$, a first-order kinetic is described; and when $\alpha>1$, a variety of sigmoidal profiles is produced.

It should be noted that the three parameter sigmoidal group of functions (such as the Logistic, Weibull, Hill, Gompertz or Richards-Chapman) would be generally acceptable solutions to fit the dose profiles individually, corresponding to a series of increasing antioxidant level. The survival Weibull distribution has been chosen for this study. However, any of the other equations mentioned are reasonably appropriate.

Apart from the previous parameters, other interesting ones can be obtained, such as the average rate $\left(v_{\tau}\right.$, average molecules of the reduced radical $/ \mu \mathrm{g}$ of $A$ ) that can be obtained with some algebraic modifications from Eq. (2) as follows:

$$
v_{\tau}=\frac{K \alpha \ln 2}{2 \tau} \quad R(A)=K \exp \left[-(\ln 2)^{1-\alpha}\left(\frac{2 v_{\tau} A}{K \alpha}\right)^{\alpha}\right]
$$

Consequently, the confidence intervals of $v_{\tau}$ could be estimated by means of Eq. (3). In addition, any given percentage $n$ of the desired response $R$ can be computed simply by rearranging Eq. (2) as follows:

$$
R(A)=K \exp \left[-\ln (1-0.01 n)\left(A / \tau_{n}\right)^{\alpha}\right]
$$

in which $n$ can be any value between $0-100 \%$, consequently the corresponding $\tau_{n}$ can be computed to obtain any $n$ percentage of the maximum radical molecules reduced of $K$. Other parameters of Eq. (4) remain with the same meaning as in Eq. (2).

Consequently, the parameter $\tau$ or $v_{\tau}$ can be considered as a meaningful way to compare $A$ activities. The information provided by the combination of these values represents a robust tool to compare the activities of different antioxidant agents based on the parametric estimations of the dose-response effects. Authors may only focus on one parameter, depending on their interests; in any case, they are a simple way of summarizing responses in useful values to compare the AC of compounds. Therefore, the potential equivalent capacity of samples and standard antioxidants can be compared 
effortlessly. Furthermore, the application may facilitate the ranking process and the selection of appropriate concentrations of natural products to replace commercial antioxidants.

\subsubsection{Classical interactive hypothesis: Identification of the mode of interaction for SET assays}

The synergistic and antagonistic interactions of two (or more) antioxidants, despite their importance, are only studied from simplistic views (Jia et al. 1998; Marinova et al. 2008; Yang et al. 2009), rather than generalizing the classical approaches (Berenbaum 1985a, 1985b; Bliss 1937, 1939; Greco et al. 1995; Loewe and Muischnek 1926). The characterization of the combined action of chemicals of interest involves the challenge of how to define the null, antagonistic or synergistic action. It is therefore of crucial importance to understand the terminology that describes the combined effect of agents in terms of the mechanisms of action (Hertzberg and MacDonell 2002). For the determination of the presence or absence of interactive effects of two well defined agents, two classical concepts or modes of interaction are conventionally considered in the dose-response field, "simple dissimilar action" or the independent action (IA) (Bliss 1939) and "simple similar action" or the concentration addition (CA) (Berenbaum 1985a, 1985b; Loewe and Muischnek 1926), also referred to as Bliss independence and Loewe additivity. Both approaches have multiple names in the literature.

The theoretical principle behind CA is that in the absence of interactions, chemicals differ only in potency and can be regarded as dilutions of one another (Berenbaum 1985a, 1985b). Because different dilutions of the same chemical will always conform to CA and will operate by the same mechanisms of action, it is typically assumed that mixtures of different chemicals that share the same molecular target will also behave according to CA. To calculate the joint effect of chemicals using $\mathrm{CA}$, one accounts for the degree of dilution by relating the concentration of each chemical in the mixture as a single agent, adding up the concentrations, and calculating the effect from the joint dose-response curve.

The theoretical assumption behind IA supposes that the compound's effects act through different mechanisms; the chemicals in a mixture do not physically, chemically, or biologically interact. Therefore, they act independently of each other (Bliss 1937; Hewlett and Plackett 1964). Berenbaum (1985a, 1985b) illustrated the theory with a hypothetical example and defined, through probability theory, the response as the sum of the probabilities of the individual phenomenon minus the probability of their joint occurrence.

IA and CA hypotheses postulate modes of action; that is, they can be associated to general mechanisms or microscopic conditions that allow variations, capable of generating specific responses. Both are useful simplifications, but do not represent the only possible responses, and avoid to a large extent the analysis of the interactions that are possible in the system.

Conventionally, the results of dose-response experiments are tested against these two hypotheses. When comparing the CA and IA hypotheses, even when the models are challenged with chemicals having different mechanisms of action and chemicals mixed according to their potency to exert equal effects, the difference in prediction by IA and CA is small. This relatively minor difference suggests that both models may be valid and researchers have to use additional statistical tests to deduce which is the most relevant solution for a particular system. From a practical point of view, it is desirable to be able to use a single model for all situations. In addition, mechanisms of action are often unknown. In this regard, toxicology and ecotoxicology fields have become the focus for non-lethal end points (Cedergreen et al. 2008). These tests measure gradual end points such as growth, morphological and behavioral changes which are all quantitative and therefore do not fit the theoretical assumptions of IA (Faust et al. 2003; Gessner 1988). The SET test fits the description of non-lethal end points 
because SET measures the reduction of only one radical to its "non-lethal" end point. Therefore, in contrast to HAT assays, in which the hypothetical assumptions involve the analysis of both possible mechanisms of action (Prieto et al. 2014b; Prieto and Vázquez 2014), SET assays can simplify the analysis using only the CA theoretical formulation.

\subsubsection{Response surface model to describe and quantify the interactive concentration addition (CA) mode of action in SET assays}

The classical null interaction formulation of CA (Berenbaum 1985a, 1985b) is not defined as a relation between the individual responses, but through the assumption that the response to a mixed dose of two chemicals behaves as the response to the "mixed" dose of the same chemical, implying that any chemical concentration can be substituted by the effective concentration of the other one. However, the conventional analysis applied in toxicology to CA hypothesis, instead of analyzing the results through conventional dose-responses tools, uses a graphical analysis with the isobole examination, accepting that straight, concave up and convex up isoboles indicate a null interaction, synergy and antagonism, respectively. Unfortunately, this graphical procedure is more a restriction than a simplification because: 1) other effects may increase or decrease the response corresponding to the null interaction, without altering the effective concentration, and these effects should not be excluded from the synergy and antagonism definitions; 2) such an index is calculated at a specific point or along a specific response (e.g. the half-maximal response), and cannot account for what happens in another region of the response surface. Therefore, the quantification of synergy and antagonism concepts will be used according to current modern approaches applying response surface models, in which the possible patterns of deviation from the reference are assessed based on dosedependent interactions (Greco et al. 1995; Hadrup et al. 2013; Jonker et al. 2005). Essentially a doseresponse relationship for each chemical applied separately is combined with a functional relationship between the concentrations of the individual chemicals in the mixture, and the single-chemical concentrations needed to obtain the same effect.

The importance of the assumptions of CA concerning both the degree of similarity of site of action and the similarity of slope has been debated since the introduction of the model (Cedergreen et al. 2008; Hertzberg and MacDonell 2002; Jonker et al. 2005; Sørensen et al. 2007). The response to a mixed dose of two antioxidant agents $\left(A_{1}\right.$ and $\left.A_{2}\right)$ in the absence of interactive effects (null interaction) can be postulated as the response of two fictitious "mixed" doses of the same agent, as described by (Murado and Prieto 2013a) as follows:

$$
R\left(A_{1}, A_{2}\right)=W\left[\left(A_{1}+A_{2}\right) ; K, m, \alpha\right]
$$

The different potency effects of each antioxidant compound tested, can be inserted by multiplying one of the doses by a factor $p$ ( $p=1$ for chemicals with equal potency), as follows:

$$
R\left(A_{1}, A_{2}\right)=W\left[\left(p A_{1}+A_{2}\right) ; K, m, \alpha\right] \text { or reciprocally } R\left(A_{1}, A_{2}\right)=W\left[\left(A_{1}+p A_{2}\right) ; K, m, \alpha\right]
$$

The $p$ coefficient ( $p>1$ if the first effector has more potency than the second one) means that if a joint response is described by Eq. (6), the $m_{2}$ parameter of the individual response to the second antioxidant agent is $m_{2}=m \times p$.

The above model does not include the interactions. The concept "interaction" describes the combined effect between two chemicals. The term "interaction" should not be viewed in the physiological 
sense to describe biological interference for a target or receptor, but as an empirical description to characterize departure from additivity. Different possible perturbations can be postulated: (1) dose dependent interactions of one antioxidant modifying the effective dose of the $A$ power and introducing a factor that perturbs it; and (2) interactions that modify the parameters of the response to the other parameters and therefore, changing the response, which can be achieved by multiplying $K$ and $m$ by a perturbation term. For simplification, in both cases, the factor that perturbs the dose or the parameters can be described by the following relationship:

$v_{\theta i}=\left(1+b_{\theta i} A_{j}\right) /\left(1+c_{\theta i} A_{j}\right) ; \quad(\mathrm{i} \neq \mathrm{j})$

where the subscript $i$ identifies the $A$ by the perturbed $A$ noted with $j$, $v_{\theta \mathrm{i}}$ is the factor that multiplies the $\theta$ parameter or antioxidant power of the response to $A_{\mathrm{i}}$, with fitting coefficients $b_{\theta \mathrm{i}}$ and $c_{\theta \mathrm{i}}$.

The general model for $C A$ is more complex, including interactions, modifying the effective dose and interactions, modifying the sigmoidal parameters with the perturbation term of Eq. (7) defined as follows:

$$
R\left(A_{1}, A_{2}\right)=W\left[\left(p A_{1} v_{A 1}+A_{2} v_{A 2}\right) ; K v_{k 1} v_{k 2}, m v_{m 1} v_{m 2}, \alpha\right]
$$

Eq. (8) includes all the possible theoretical interactions, but much simpler situations are normally found.

\subsubsection{Simplification of the complex interactive effects in a simple quantification index}

Once the previous relations are accepted as a generalized model for $C A$ hypothesis, an algebraic framework is established that characterizes synergy and antagonism scenarios through the specific variations imposed by the perturbations, translated into changes of the effective concentrations as well as into variations of $K$ and $m$. However, all these complex possible effects described above may obstruct the comprehension of the overall effects accounted, and a single numerical value that summarizes the nature and the intensity of the synergistic or antagonistic interactions could be very helpful. The best alternative would be to summarize the complex possible effects described above by computing the percentage relative unit of volume $(R U V)$ between the volume of the surface produced by the null interaction $\left(S V_{N I}\right)$ and the volume of the surface with interactions $\left(S V_{I}\right)$ as follows:

$$
R U V=\frac{S V_{I}-S V_{N I}}{S V_{I}} \times 100 ; \text { being } S V=h_{i} h_{j} \sum_{i=0}^{n} \sum_{j=0}^{m} f\left(A_{i} A_{j}\right) \phi_{i, j}
$$

in which $A_{\mathrm{i}}$ and $A_{\mathrm{j}}$ are the dependent variables that represent the $n$ and $m$ concentration of both antioxidants, $h_{\mathrm{i}}$ and $h_{\mathrm{j}}$ are the concentration interval sets and $\Phi_{\mathrm{i}, \mathrm{j}}$ is the product of the nested composite trapezoidal rule coefficients. Therefore, positive and negative values of $R U V$ describe the predominantly synergistic and antagonistic interaction effects between the antioxidants over the study range. This index summarizes the effect produced, but changes proportionally as the concentration ranges change; this can serve as a guiding value, which at least is more informative than providing a proportional number of arrows up and down.

\subsubsection{Experimental design for the combination of two antioxidant agents, number of concentrations and ranges more suitable for efficient analysis}


When analyzing the individual dose effects of one agent with dose-response models (Murado and Prieto 2013b), the focus should be on the experimental effort covering a range, rather than increasing the number of replicates to minimize the effects of the experimental error, because from the point of statistical significance of a model, this is more efficient. For increasing the accuracy of the model predictions and significance of the parameter estimations, assuming a standard deviation of a $5 \%$ with a homoscedastic variance of the experimental error, we must perform at least 12 proportional independent measures through the independent variable, with a minimum of $25 \%$ of the responses on the asymptote. As described (Murado and Prieto 2013b), by following these simple rules, we ensure (with a $95 \%$ of likelihood) that all the parametric estimations obtained would be statistically consistent with an error less than $5 \%$. To obtain the concentration ranges that would produce $25 \%$ of the dose-responses in the asymptote, initial minor dose-range experiments must be performed. The final ranges used for the individual agents for the SET assays illustrated in this study were all clarified in the material and methods section.

When analyzing the combining dose effects of two agents with a dose-response surface model, the essence of the individual agent analysis must be kept, but the multi-component variables affecting the efficiency of the mathematical analysis are difficult to distinguish. To accurately quantify, characterize and predict the additive behavior of compounds as null, synergistic or antagonistic in SET assays, the response would heavily depend on appropriate study designs. From this starting point, one might speculate how the compounds would behave in a mixture in acute, single-dose experiments. However, these are simplistic reductions of the problem (Groten 2000). The only way to describe the combined action of the components in the mixture is to perform experimental studies combining the doses and comparing the effect of the mixture to the effect of the individual compounds (Murado and Prieto 2013a). In principle, there is a diverse range of experimental designs. Each design offers specific advantages, but in our experience, the complete design is the most effective, which is simply combining all the doses of an agent with all the doses of the other. Although the complete design is more informative and efficient than the radial or equiadditive designs (Sørensen et al. 2007), commonly used in the risk assessment of toxic chemicals, its drawback is that it requires an additional set of data compared to the other designs. Regarding the number of doses that should be combined, we have fulfilled the maximum combined area of a typical 96 well microplate, in which an array of eight independent doses per each antioxidant, are combined, providing 64 independent dose relationships.

When we fit the experimental data to a multivariable characterization, the model becomes more consistent and informative, as well as providing better estimations of parameters reducing their confidence interval. In as much as the experimental curves obtained do not span the full range and some of them fail to provide information about one or more of the parameters of the equation, the combined application of a surface model would describe simply and accurately all the responses. Finally, regarding the concentration ranges to be used, we strongly suggest that the ideal dose range in the combined experiment should be those that produce $95 \%$ of the response in the initial analysis of the individual effects. Such an ideal range of doses can be determined easily by firstly fitting the dose-response of the individual effects to Eq. (2), and then, inserting the parametric estimations obtained into Eq. (4) with $\mathrm{n}=95 \%$.

\subsubsection{Number of antioxidants and type (individual or mixtures) that theoretically are subjected to analysis}

When assessing the toxicity of chemicals over the life of organisms, researchers argue that they are rarely exposed to only the combination of two single contaminants. Such arguments may be equally 
valid in the analysis of joint effects of antioxidants. Although several studies in the literature have illustrated procedures to assess a multi-component mixture of individual chemical entities (Faust et al. 2003), we have only discussed binary mixtures in this study. Experiments with three or more chemicals do not seem to have a satisfactory resolution (Murado and Prieto 2013a; Sørensen et al. 2007).

With regard to the type of antioxidants that theoretically are valid in order to test their combined effects, once the AC hypothesis is established as a general solution for the SET assays, the following binary combinations are acceptable: (1) two individual chemical entities, and (2) an individual chemical entity with a mixture of unknown chemical entities (such as complex antioxidant extracts). In the first one, we would establish the effects of its combined response and some possible conjectures of their mechanistic interaction could be drawn. In the second one, even if the combination of similar or dissimilar individual chemical entities with antioxidant extracts, formed by a mixture of agents, is at least controversial, we would still be able to determine general effects of its joint action. To our knowledge, for testing mixtures of unknown compounds, no tools or hypotheses have been developed. However, we could treat the extracted residues as a mixture of compounds and test their collective joint action response as if they are well-defined chemical entities.

The responses to the binary joint interaction of pure antioxidants (such as BHT, ETX, TRO) and the mixture of antioxidant compounds from different chemical entities (extracted residues), described in the material and methods section were used to exemplify the developed procedure.

\subsection{Application to assess and compare the potential AC as a function of a dose-response analysis}

The previous standardizations (Eq. (1)) and mathematical dose-response modeling (Eq. (2) and (3)) were applied to the standard antioxidants and extracts described in the material and methods section in both experimental reactions (DPPH and ABTS). Figure 1 (part A and B) shows the graphical analysis to all the standard antioxidants and extracts for the DPPH and ABTS assays, respectively. The dots $(\mathrm{O})$ are the standardized values and the lines the fittings to Eq. (2) for all the tested agents. To simplify the comparison process for both reactions, the dose-responses of extracts and the commercial antioxidants are expressed in $\mu \mathrm{g}$ of the compound. Regarding the $\mathrm{AC}$ of antioxidant extracts ( $\mathrm{C}$ and $\mathrm{T})$, this shows a clear dose-time dependency for the two SET assays tested here. The fitting parameters, the parametric statistical estimations and correlation coefficients of determination $\left(r^{2}\right)$ are presented in Table 1. Finally, Figure 1 (part C) shows the numerical values of parameter $v_{\tau}$ (Eq. (3)) as assessment criteria to compare their potential AC.

In general, the quantity of antioxidant needed, to counteract the DPPH radicals is less than that required to counteract the ABTS radical. Accurate dose-responses are obtained in all cases. The following order of activities can be established for each of the reactions:

-For DPPH the antioxidant potential would be: $\mathrm{PG}>\mathrm{AA}>\mathrm{TBHQ}>\mathrm{T}>\mathrm{TRO}>\mathrm{BHA}>\mathrm{ETX}>\mathrm{TOC}$ $>\mathrm{C}>\mathrm{BHT}$.

-For ABTS the antioxidant potential would be: $\mathrm{PG}>\mathrm{TBHQ}>\mathrm{ETX}>\mathrm{BHA}>\mathrm{T}>\mathrm{TRO}>\mathrm{TOC}>\mathrm{C}>$ $\mathrm{AA}>\mathrm{BHT}$.

Beyond quantitative differences, the analysis of the parametric non-linear response of the antioxidant equivalent action and rigorous comparison of their capacity provides a tool that facilitates the selection of appropriate concentrations of products to replace commercial antioxidants. Thus, the 
potential equivalent capacity can be computed easily. For example, the following in vitro results can be concluded:

-In DPPH environments, $\sim 2 \mu \mathrm{g}$ of $\mathrm{T}$ is equivalent to $\sim 1 \mu \mathrm{g}$ of PG.

-In ABTS environments, $\sim 2.51 \mu \mathrm{g}$ of $\mathrm{C}$ is equivalent to $\sim 1 \mu \mathrm{g}$ of TRO.

When the analyses are based on the $v_{\tau}$ parameter, in some cases the equivalent values are greater than some of the standard antioxidants.

\subsection{Null, synergistic and antagonistic analysis of the joint effect of compounds}

\subsubsection{Illustration example of the methodological process}

The methodological procedure and the mathematical models proposed in the previous sections yielded consistent results when combining all the antioxidants listed in the materials section for each of the methods. These results not only permitted the decision between the null interaction, synergy and antagonism, but also revealed some interesting aspects of the system reactions. To illustrate the methodological procedure of this approach, the joint action of AA and TBHQ on the reduction of the radical form of DPPH is now described in detail.

Figure 2 (plot A) shows the application of the null interaction hypothesis of the joint action, described by adjusting the surface-response to Eq. (6). By proceeding in this way, the $r^{2}$ and $R_{a d j}^{2}$ values, as well as the Student's $t$ and Fisher's $F$ test (both with $\alpha=0.05$ ) applied to the parametric estimations and to the explained variance, respectively, showed a statistically acceptable fit (Figure 2, part A). However, the distribution between the observed and predicted results was biased, and the residuals showed that the computed response surface predicts higher values than those experimentally obtained, which suggests an antagonistic interaction. When we assume the interactive hypothesis (Figure 2, plot B), as described by Eq. (8), a decrease in the bias and an improvement in the other fitting criteria were obtained by accepting an increase in the $m$ parameter of the response to TBHQ due to the presence of AA (increasing antioxidant potency: antagonistic in the strict sense). A further improvement could be obtained by accepting a similar drop in the $K$ parameter (antagonism in the broad sense). All fitting criteria improved significantly when those antagonistic effects were included in comparison to those shown by assuming no interaction in Eq. (6). Therefore, it must be concluded that the effect of the joint response to AA and TBHQ shows an antagonistic interaction.

When a large set of data needs to be analyzed, the intuitive process of finding the most appropriate solution can be very laborious. To overcome this, we have developed a routine in excel in which all possible parameter combinations were tested to rank and select the most appropriate solution (see material and methods section). The selection was identical to that intuitively found above, which demonstrates the reliability of both options for selecting the correct solution. However, because the automatic system is undoubtedly faster and more reliable, it was the procedure used to assess all subsequent pairs of tested antioxidants.

Once the modeling of the experimental surface responses is determined, we could quantify the degree of interactive effect (\%RUV) as described in Eq. (9). The variations in the parametric values of the response to an antioxidant as a function of the concentration of the other antioxidant (the structures of the perturbation terms) or the global approach of computing the $R U V$ allow a brief reasonable description of the interactive effects. The synergistic and antagonistic consequences can 
vary along the response surface, producing effects with opposite signs in different subdomains of the surface.

Therefore, the datum of practical interest is the possible difference between the null interaction and the experimental result in a given domain. Only the "scenery" of these differences throughout the experimental domain allows effective and statistically sound statements. Finally, Figure 2 (plot C) presents the obtained \% $R U V$ data, first in two separate 2D graphs that show the response for the individual effects caused for each antioxidant, and then as the response and antioxidant doses presented in a single 3D graph.

\subsubsection{Joint action between several pairs of antioxidant standards}

By using the standard antioxidants listed in the materials section, 36 combinations were performed for each SET assay, including those in which the pair of antioxidant is the same antioxidant (used simply as a control). Each pair of agents displays 64 concentration combinations in terms of its maximum potential activity as described in the materials section. All binary agent responses are subjected to the automatic stepwise regression analysis described in the numerical methods section, which provides the information regarding the mode of interaction by applying Eq. (6) and (8), the $C A$ hypothesis without and with interactions, respectively. The full analysis of all the possible combinations is presented in the appendix (Table A1 and Table A2). For simplicity, only a representative set of 21 case combinations were chosen and presented in Figure 3 and Figure 4.

All the adjusted coefficients of determination $R_{a d j}^{2}$ of all fitting solutions were always greater than 0.97 , with a wide majority of the fittings superior at 0.99 . Some exceptional cases, if their behaviour is described and confirmed by other areas of study, may show some interesting concepts for the field of the interactive effects of compounds.

\subsubsection{Joint action of several pairs of compounds}

The interactive effects of the extracts from different antioxidant extracts were tested between them and against the other compounds. Figure 5 shows the matrix combination of 16 binary agents for each SET assay, including those in which each antioxidant in a pair is identical (used simply as a control). The modes of interaction, parametric estimations, confidence intervals and statistical information of best fitting results derived for each of the binary combinations tested are presented in Table A3 (appendix section). The adjusted coefficients of determination $R_{a d j}^{2}$ of all fitting solutions were always greater than 0.97 , with a wide majority of the fittings superior at 0.99 . To our knowledge, no tools or hypotheses have been developed for testing mixtures of compounds. Thus, we treat the extracted residues as a mixture of compounds. In any case, the responses to the binary joint interaction of commercial antioxidants (such as BHT, ETX, TRO) and the mixture of antioxidant compounds from different chemical entities (extracted residues) produced consistent results in all cases.

Once the mode of interaction is identified, the quantification of the interactive effects is determined in terms of $R U V$ by means of Eq. (9).

\subsection{Reproducibility of the methodology and confidence limit of RUV determination}

The reproducibility and limit of confidence of the methodological procedure was tested by analyzing all the standard antioxidants several times and determining the confidence interval of the $\% R U V$ 
result response for each SET assay. The results can be observed in Figure 6. For the ABTS assay, the global confidence interval was $\pm 1.95 \%$, while for the DPPH reaction it was $\pm 2.01 \%$. As a general rule for both assays, it can be established that any $\% R U V$ value between -2.00 to $2.00 \%$ cannot be considered as statistically consistent. Therefore, based on this limit of confidence for the $R U V$ values obtained, the statistically consistent responses determined from the mixtures tested are displayed in Table 2 .

In terms of the type of interactions (synergistic/antagonistic/null interaction) for the DPPH assay; four cases were found with significant antagonistic responses, the TBHQ vs AA, BHA vs ETX, TOC vs AA and BHA vs TOC. Five other cases were found with significant synergistic interactions (ETX vs T, TBHQ vs T, TRO vs C, BHT vs $\mathrm{C}$ and ETX vs C). It was notable that all the antagonistic significant interactions were between individual chemical entities, while all significant synergistic interactions were between the individual chemical entities and the complex mixtures of antioxidants from the extracted residues. For the ABTS assay; ten cases were found with significant antagonistic responses, while only two were found with significant synergistic interactions. All other binary mixtures did not produce significant lower or higher responses than expected (null interaction). However, there were a few cases in which their synergistic/antagonistic effects, in both reactions, were within $\pm 2.0 \%$ of confidence limit, whereby the interactive hypothesis could possibly be admitted, due to the behaviour of the response surface difference. A deeper analysis must be carried out to confirm whether or not there is an interaction.

\section{CONCLUSIONS}

Synergy and antagonism are controversial characteristic behaviors of very diverse systems. Despite their importance, the common characterization of these phenomena in the context of the antioxidant action is often questionable due to some problematic definitions and the type of data used. In this paper, a methodological procedure has been developed for the joint action of several pairs of antioxidants, which enables the determination and quantification of the synergistic and antagonistic interactive effects. Well-established ideas from different existing fields are used to replace the current simplistic procedures to quantify the effects of joint responses. One of the common hypotheses for describing the combined effects is established for SET assays. A dose dependent mathematical model representative of that hypothesis, based on probability functions with meaningful parameters, is applied. The interactive effects between antioxidants are introduced into the model with simple auxiliary functions that describe the variations induced by each antioxidant in the parameters that define the effects of the other. Finally, a comprehensive index to summarize the complex parametric responses in one single value is proposed. Although the approach was experimentally demonstrated just in two classical SET assays (DPPH and ABTS), the results are directly expandable to other types of classical SET assays. Statistically consistent responses of null, synergy and antagonism effects were found when characterizing the interactions between several pairs of individual and complex mixtures of chemical antioxidant agents.

Two types of antioxidant samples, individual chemical entities and complex mixtures, covering as much as possible the range of possible responses, were used to test the rapid methodology proposed here. The results shows that the AC of the extracts was more similar compared to some of the commercial standards in terms of potential maximum capacity, while others showed lower values. The proposed generalized procedures for the joint action of several well-known antioxidants produced consistent results in all cases.

Afterwards, we determined and quantified the null/synergistic/antagonistic interactions between binary combinations of those types of antioxidants using the developed methodological procedure. 
Therefore, in the assessment of the possible interactive effects in binary mixtures of antioxidants, the new procedure represents a powerful informative tool, which is effectively designed for defining the important aspects of very complex responses. Although the proposed approach is a little more complicated than some relatively common solutions, we believe that it is free from the most controversial aspects of such approaches.

\section{ACKNOWLEDGEMENTS}

Miguel Ángel Prieto Lage was awarded with one travelling grant provided by the Axencia Galega de Innovación (Xunta Galicia) and the European Social Fund (ESF). Last but not least, the authors want to express their gratitude to Araceli Menduiña Santomé for her professional work and dedication. 


\section{FIGURE CAPTIONS}

Figure 1: Parts A and $\mathbf{B}$ show the illustrative application of the model developed (Eq. (2)) to analyze the dose-effect of the antioxidant responses (standards and extracts) for the DPPH and ABTS assay, respectively. The dots $(O)$ are the experimental data series and the lines the fittings. Part $\mathbf{C}$ shows the numerical values of the parameter $v_{\tau}$ of Eq. (3) as assessment criteria. Parametric values of the fittings are presented in Table 1 and dose ranges in material and methods section.

Figure 2: An illustrative procedure to obtain the $R A U$ responses using the AA and TBHQ antioxidant combination in the DPPH reaction as an example. The final RUV value of such interaction was $-4.94 \%$, more details about the fittings in Table 1 and Table A1.

Figure 3: Matrix combination responses for the DPPH assay, which is organized as follows: a) the results obtained for the controls can be seen in the diagonal; b) in the top part of the diagonal the surface responses for each pair antioxidant combination is presented; and c) in the bottom diagonal part, the different "scenery" between their respective null interaction form and the obtained response is presented. Numerical results are in Table 2 and Table A1.

Figure 4: Matrix combination responses for the ABTS assay, which is organized as follows: a) in the diagonal, the results obtained for the controls it can be seen; b) in the top part of the diagonal, the surface responses for each pair antioxidant combination is presented; and c) in the bottom diagonal part, the different "scenery" between their respective null interaction form and the obtained response is presented. Numerical results are in Table 2 and Table A2.

Figure 5: Combination responses for the antioxidant extracts against standard antioxidants in the DPPH and ABTS reactions. To surfaces response graphs for each case for each reaction are show: one belongs to results obtained for the joint response and the other to the differences "scenery" between their respective null interaction form and the obtained response. Numerical results are in Table 2 and Table A3.

Figure 6: Reproducibility of the methodological procedure and limit of confidence for the $\% R U V$ results. Note that the range of axis $\mathrm{z}$ is six times lower than those presented in Figure 3, Figure 4 and Figure 5. 


\section{TABLE CAPTIONS}

Table 1: Parametric estimations and statistic information of the kinetic series of the dose-responses of all antioxidant standards and extracts fitted to the kinetic Eq. (2), (3) and (4).

Table 2: Effect of the combination of 45 different pairs of standard antioxidants and extracts for each SET reaction. For those cases where each sample is combined with itself, the results are used simply as a control. For each statistically consistent case the resulting $R U V$ values are presented (ns if $R U V$ $<2.0 \%$, see text for more details). The concentration ranges used for each case are shown in $\mu \mathrm{g}$ for the final reaction volume of the reaction $(300 \mu \mathrm{L})$.

\section{APPENDIX TABLE CAPTIONS}

Table A1: Parametric values of the joint action between different standard antioxidants in the DPPH reaction by fitting the experimental results to Eq. (6) (assuming no interactions) and (8) (assuming interactive mechanisms). The degree of interactive effect ( $\% R U V)$ is computed as described in Eq. (9). For all the presented parameters, their estimations are significant.

Table A2: Parametric values of the joint action between different standard antioxidants in the ABTS reaction by fitting the experimental results to Eq. (6) (assuming no interactions) and (8) (assuming interactive mechanisms). The degree of interactive effect $(\% R U V)$ is computed as described in Eq. (9). For all the presented parameters, their estimations are significant.

Table A3: Parametric values of the joint action of different standard antioxidants against two natural extracts (T and $\mathrm{C}$ ) in the DPPH and ABTS reaction by fitting the experimental results to Eq. (6) (assuming no interactions) and (8) (assuming interactive mechanisms). The degree of interactive effect $(\% R U V)$ is computed as described in Eq. (9). For all the presented parameters, their estimations are significant. 


\section{REFERENCES}

Almajano, M. P., R. Carbó, M. E. Delgado, and M. H. Gordon. 2007. "Effect of pH on the Antimicrobial Activity and Oxidative Stability of Oil-in-Water Emulsions Containing Caffeic Acid." Journal of food science 72(5):C258-263.

Apak, Reşat et al. 2013. "Methods of Measurement and Evaluation of Natural Antioxidant Capacity/activity (IUPAC Technical Report).” Pure and Applied Chemistry 85(5):957-98.

Arts, M. 2004. "A New Approach to Assess the Total Antioxidant Capacity Using the TEAC Assay." Food Chemistry 88(4):567-70.

Berenbaum, M. C. 1985a. "Consequences of Synergy between Environmental Carcinogens." Environmental research 38(2):310-18.

Berenbaum, M. C. 1985b. "The Expected Effect of a Combination of Agents: The General Solution." Journal of theoretical biology 114(3):413-31.

Bliss, C. I. 1937. "The Calculation of the Time-Mortality Curve." Annals of Applied Biology 24:81524.

Bliss, C. I. 1939. "The Toxicity of Poisons Applied Jointly.” Annals of Applied Biology 26(3):585615.

Bruun-Jensena, Lone, and IM Skovgaardb. 1994. "Antioxidant Synergism between Tocopherols and Ascorbyl Palmitate in Cooked, Minced Turkey." Zeitschrift für Lebensmittel-Untersuchung und Forschung 199(3):210-13.

Cedergreen, N. et al. 2008. "A Review of Independent Action Compared to Concentration Addition as Reference Models for Mixtures of Compounds with Different Molecular Target Sites."

Environmental 27(7):1621-32.

Faust, M. et al. 2003. "Joint Algal Toxicity of 16 Dissimilarly Acting Chemicals Is Predictable by the Concept of Independent Action." Aquatic Toxicology 63(1):43-63.

Floegel, Anna, Dae-Ok Kim, Sang-Jin Chung, Sung I. Koo, and Ock K. Chun. 2011. "Comparison of ABTS/DPPH Assays to Measure Antioxidant Capacity in Popular Antioxidant-Rich US Foods." Journal of Food Composition and Analysis 24(7):1043-48.

Frankel, E. N., and J. W. Finley. 2008. "How to Standardize the Multiplicity of Methods to Evaluate Natural Antioxidants." Journal of Agricultural and Food Chemestry 56(13):4901-8.

Gessner, P. K. 1988. “A Straightforward Method for the Study of Drug Interactions: An Isobolographic Analysis Primer.” International Journal of Toxicology 7(7):987-1012.

Gieseg, S. P., and H. Esterbauer. 1994. "Low Density Lipoprotein Is Saturable by pro-Oxidant Copper." FEBS letters 343(3):188-94. 
Greco, W. R., G. Bravo, and J. C. Parsons. 1995. "The Search for Synergy: A Critical Review from a Response Surface Perspective.” Pharmacological reviews 47(2):331-85.

Groten, J. P. 2000. "Mixtures and Interactions." Food and chemical toxicology : an international journal published for the British Industrial Biological Research Association 38(1 Suppl):S65-71.

Hadrup, Niels et al. 2013. "Concentration Addition, Independent Action and Generalized Concentration Addition Models for Mixture Effect Prediction of Sex Hormone Synthesis in Vitro." PloS one 8(8):e70490.

Hertzberg, Richard C., and Margaret M. MacDonell. 2002. "Synergy and Other Ineffective Mixture Risk Definitions." The Science of the total environment 288(1-2):31-42.

Hewlett, PS, and R. L. Plackett. 1964. "A Unified Theory for Quantal Responses to Mixtures of Drugs: Competitive Action.” Biometrics 20(3):566-75.

Huang, D., B. Ou, R. L. Prior, and L. Ronald. 2005. "The Chemistry behind Antioxidant Capacity Assays." Journal of Agricultural and Food Chemestry 53(6):1841-56.

Jia, Z. S., Bo Zhou, Li Yang, L. M. Wu, and Z. L. Liu. 1998. “Antioxidant Synergism of Tea Polyphenols and Tocopherol against Free Radical Induced Peroxidation of Linoleic Acid in Solution." J. Chem. Soc., Perkin Trans. 2 1(4):911-16.

Jiménez-Escrig, Antonio, Isabel Jiménez-Jiménez, Concepción Sánchez-Moreno, and Fulgencio Saura-Calixto. 2000. "Evaluation of Free Radical Scavenging of Dietary Carotenoids by the Stable Radical 2,2-Diphenyl-1-Picrylhydrazyl.” Journal of the Science of Food and Agriculture 80(11):1686-90.

Jonker, Martijs J., Claus Svendsen, Jacques J. M. Bedaux, Marina Bongers, and Jan E. Kammenga. 2005. "Significance Testing of Synergistic/antagonistic, Dose Level-Dependent, or Dose RatioDependent Effects in Mixture Dose-Response Analysis." Environmental toxicology and chemistry 24(10):2701-13.

Loewe, S., and H. Muischnek. 1926. "Über Kombinationswirkungen.” Naunyn-Schmiedebergs Archiv für experimentelle Pathologie und Pharmakologie 114(5-6):313-26.

Marinova, E., A. Toneva, and N. Yanishlieva. 2008. "Synergistic Antioxidant Effect of [alpha]Tocopherol and Myricetin on the Autoxidation of Triacylglycerols of Sunflower Oil." Food Chemistry 106(2):628-33.

Murado, M. A., and M. A. Prieto. 2013a. "Dose-Response Analysis in the Joint Action of Two Effectors. A New Approach to Simulation, Identification and Modelling of Some Basic Interactions." PLoS ONE 8(4):e61391.

Murado, M. A., and M. A. Prieto. 2013b. "NOEC and LOEC as Merely Concessive Expedients: Two Unambiguous Alternatives and Some Criteria to Maximize the Efficiency of Dose-response Experimental Designs.” Science of The Total Environment 461-462:576-86. 
Murado, M. A., and J. A. Vázquez. 2010. "Mathematical Model for the Characterization and Objective Comparison of Antioxidant Activities." Journal of Agricultural and Food Chemestry 58(3):1622-29.

Nabavi, Seyed Fazel et al. 2013. "Antioxidant and Antihemolytic Activity of Lipid-Soluble Bioactive Substances in Avocado Fruits." Fruits 68(3):185-93.

Niki, Etsuo. 2010. "Assessment of Antioxidant Capacity in Vitro and in Vivo." Free radical biology \& medicine 49(4):503-15.

Obón, J. M., M. R. Castellar, J. a. Cascales, and J. a. Fernández-López. 2005. “Assessment of the TEAC Method for Determining the Antioxidant Capacity of Synthetic Red Food Colorants." Food Research International 38(8-9):843-45. Retrieved June 15, 2011

Ozgen, Mustafa, R. Neil Reese, Artemio Z. Tulio, Joseph C. Scheerens, and a Raymond Miller. 2006. "Modified 2,2-Azino-Bis-3-Ethylbenzothiazoline-6-Sulfonic Acid (abts) Method to Measure Antioxidant Capacity of Selected Small Fruits and Comparison to Ferric Reducing Antioxidant Power (FRAP) and 2,2'-Diphenyl-1-Picrylhydrazyl (DPPH) Methods." Journal of Agricultural and Food Chemestry 54(4):1151-57.

Özilgen, S., and M. Özilgen. 1990. "Kinetic Model of Lipid Oxidation in Foods.” Journal of Food Science 55(2):498-501.

Perva-Uzunalić, Amra et al. 2006. "Extraction of Active Ingredients from Green Tea (Camellia Sinensis): Extraction Efficiency of Major Catechins and Caffeine." Food Chemistry 96(4):597-605.

Peyrat-Maillard, M. N., M. E. Cuvelier, and C. Berset. 2003. "Antioxidant Activity of Phenolic Compounds in 2, $2^{\prime}$-Azobis ( 2-Amidinopropane ) Dihydrochloride ( AAPH ) -Induced Oxidation : Synergistic and Antagonistic Effects." 80(10):1007-12.

Prieto, M. A., M. A. Murado, and J. A. Vázquez. 2014a. "A Critical Point: The Problems Associated with the Variety of Criteria to Quantify the Antioxidant Capacity." Journal of Agricultural and Food Chemestry (In press).

Prieto, M. A., M. A. Murado, and J. A. Vázquez. 2014b. "Quantification, Characterization and Description of Synergy and Antagonism in the Antioxidant Response." Food Research International 60(6):218-29.

Prieto, M. A., I. Rodríguez-Amado, J. A. Vázquez, and M. A. Murado. 2012. "B-Carotene Assay Revisited. Application to Characterize and Quantify Antioxidant and Prooxidant Activities in a Microplate." Journal of agricultural and food chemistry 60(36):8983-93.

Prieto, M. A., and J. A. Vázquez. 2014. "In Vitro Determination of the Lipophilic and Hydrophilic Antioxidant Capacity of Unroasted Coffee Bean Extracts and Their Synergistic and Antagonistic Effects." Food Research International (In press).

Prikler, Simon. 2009. "Robert de Levie: Advanced Excel for Scientific Data Analysis, 2nd Ed." Analytical and Bioanalytical Chemistry 395(7):1945. 
Re, R. et al. 1999. "Antioxidant Activity Applying an Improved ABTS Radical Cation Decolorization Assay.” Free Radical Biology and Medicine 26(9-10):1231-37.

Roginsky, V., and E. Lissi. 2005. "Review of Methods to Determine Chain-Breaking Antioxidant Activity in Food.” Food Chemistry 92(2):235-54.

Sanchez-Moreno, C. 2002. "Review: Methods Used to Evaluate the Free Radical Scavenging Activity in Foods and Biological Systems." Food Science and Technology International 8(3):12137. Retrieved July 17, 2014

Serpen, Arda, Edoardo Capuano, Vincenzo Fogliano, and Vural Gökmen. 2007. "A New Procedure to Measure the Antioxidant Activity of Insoluble Food Components." Journal of Agricultural and Food Chemestry 55(19):7676-81.

Sharma, O. P., and T. K. Bhat. 2009. "DPPH Antioxidant Assay Revisited." Food Chemistry 113(4):1202-5. Retrieved July 12, 2011

Sørensen, Helle, Nina Cedergreen, Ib M. Skovgaard, and Jens C. Streibig. 2007. "An Isobole-Based Statistical Model and Test for Synergism/antagonism in Binary Mixture Toxicity Experiments." Environmental and Ecological Statistics 14(4):383-97.

Terpinc, Petra, Miran Bezjak, and Helena Abramovič. 2009. "A Kinetic Model for Evaluation of the Antioxidant Activity of Several Rosemary Extracts." Food Chemistry 115(2):740-44.

Weibull, Waloddi, and Stockholm Sweden. 1951. "A Statistical Distribution Function of Wide Applicability." Journal of applied mechanics 18(3):293-97.

Yang, Wen-Jian et al. 2009. "Synergistic Antioxidant Activities of Eight Traditional Chinese Herb Pairs." Biological \& pharmaceutical bulletin 32(6):1021-26. 


\section{FIGURES}

A: ANTIOXIDANT DOSE-RESPONSES (STANDARDS AND SAMPLES) TO THE DPPH ASSAY
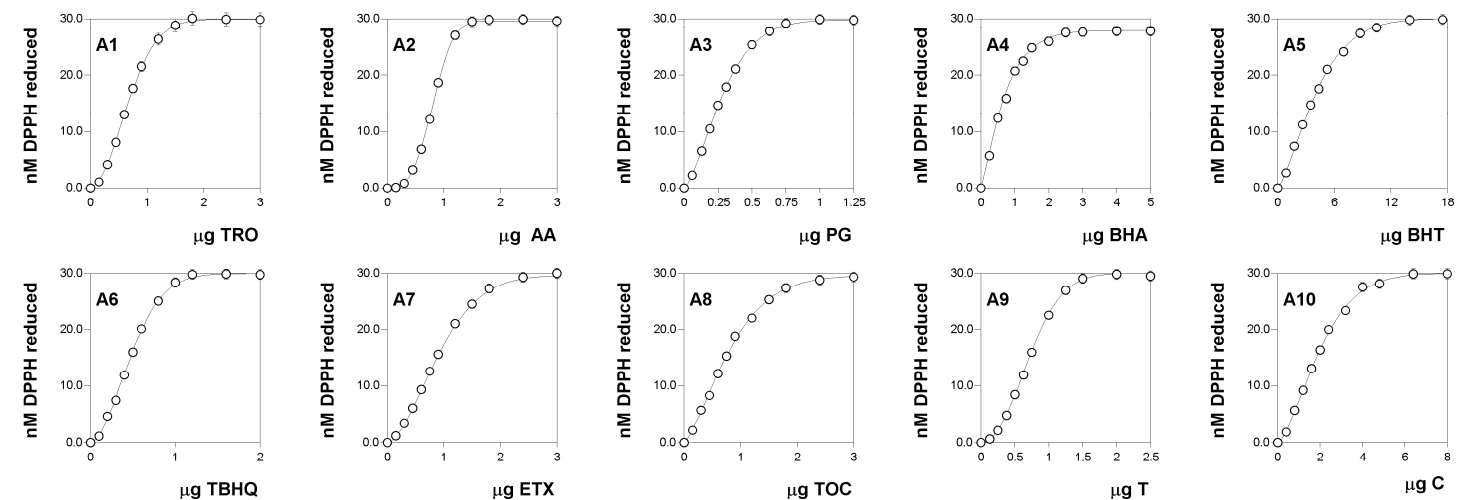

B: ANTIOXIDANT DOSE-RESPONSES (STANDARDS AND SAMPLES) TO THE ABTS ASSAY
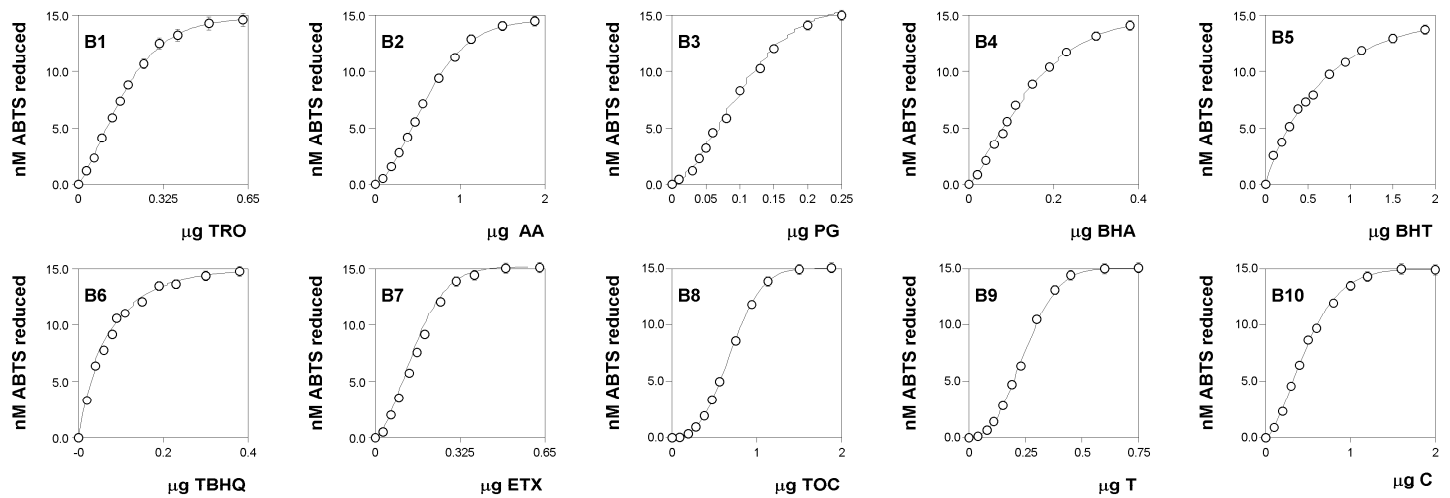

C: NUMERICAL CRITERIA VALUES FOR COMPARISON
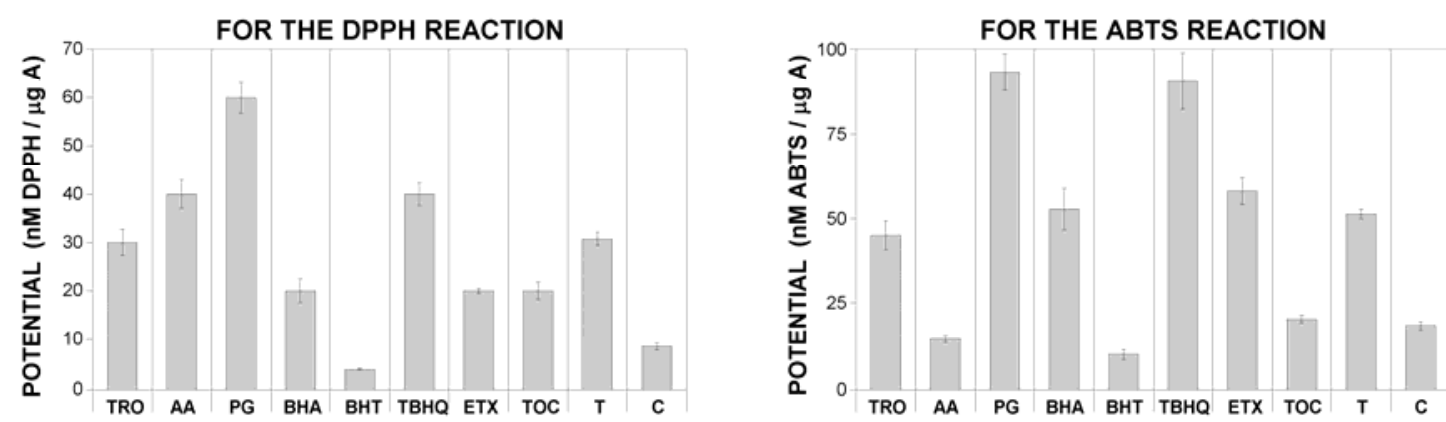

Figure 1: Parts A and $\mathbf{B}$ show the illustrative application of the model developed (Eq. (2)) to analyze the dose-effect of the antioxidant responses (standards and extracts) for the DPPH and ABTS assay, respectively. The dots $(O)$ are the experimental data series and the lines the fittings. Part $\mathbf{C}$ shows the numerical values of the parameter $v_{\tau}$ of Eq. (3) as assessment criteria. Parametric values of the fittings are presented in Table 1 and dose ranges in material and methods section. 

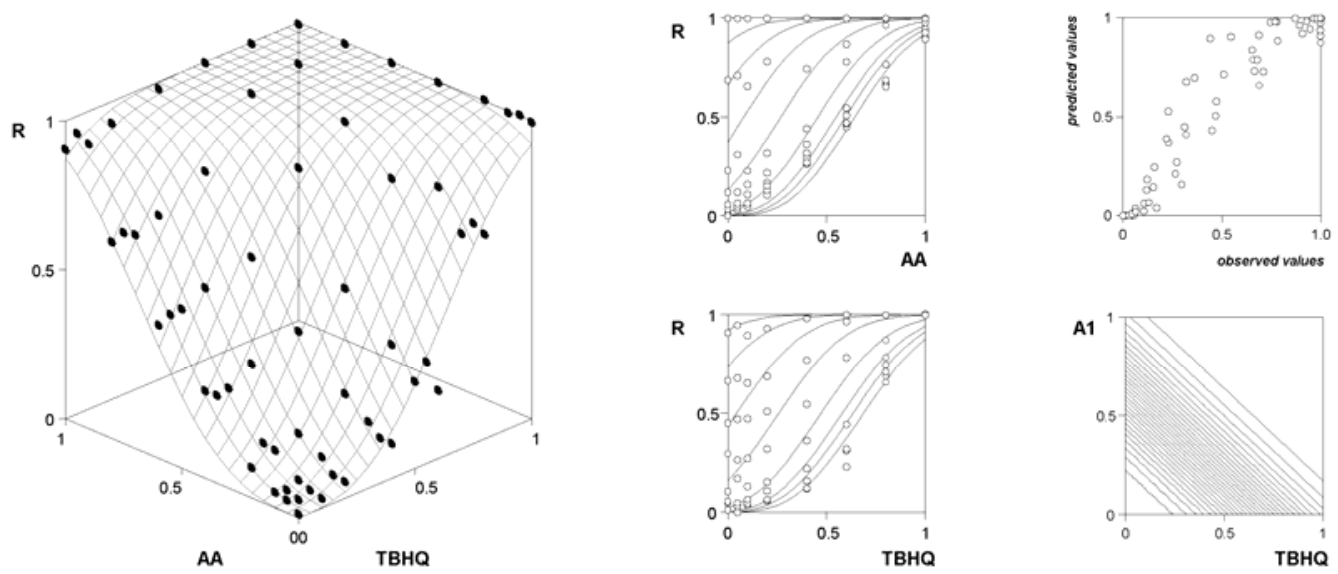

B: CONCENTRATION ADDITION WITH INTERACTIONS
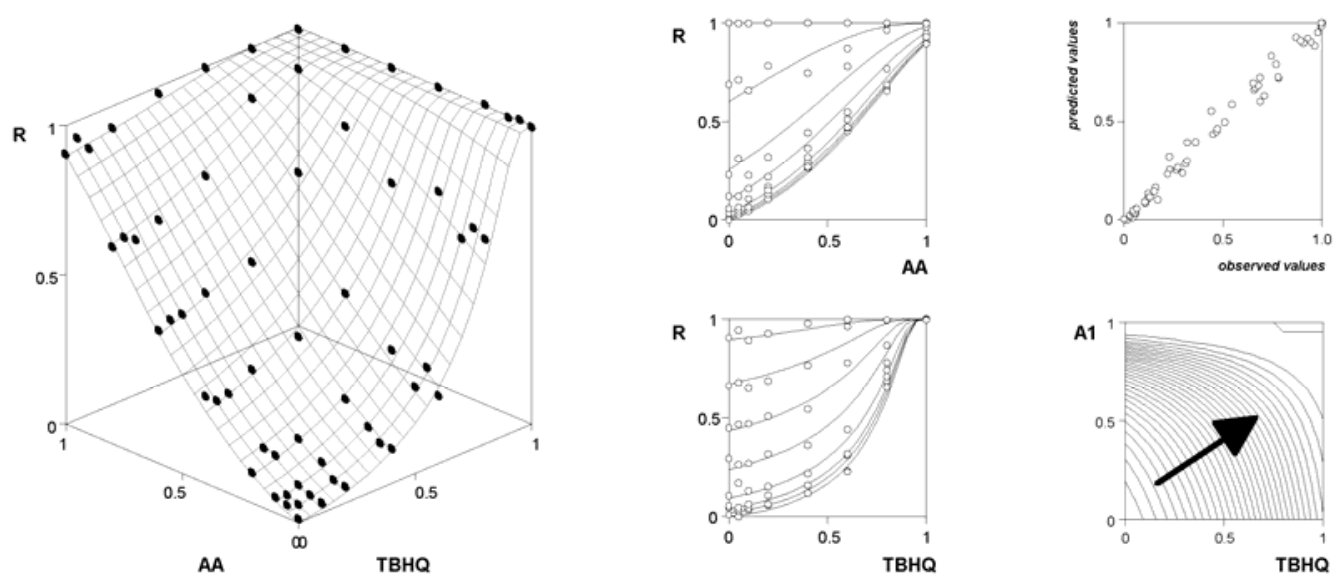

C: VOLUMETRIC DIFFERENCE BETWEEN BOTH ASSUMPTIONS
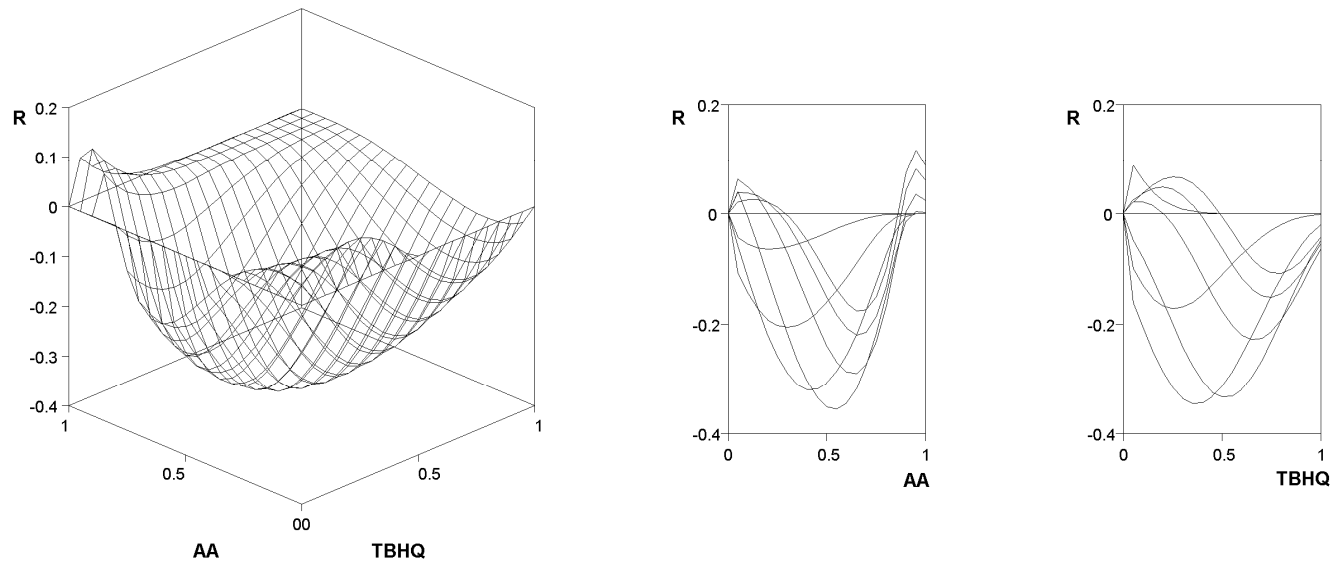

Figure 2: An illustrative procedure to obtain the $R A U$ responses using the AA and TBHQ antioxidant combination in the DPPH reaction as an example. The final RUV value of such interaction was $-4.94 \%$, more details about the fittings in Table 1 and Table A1. 


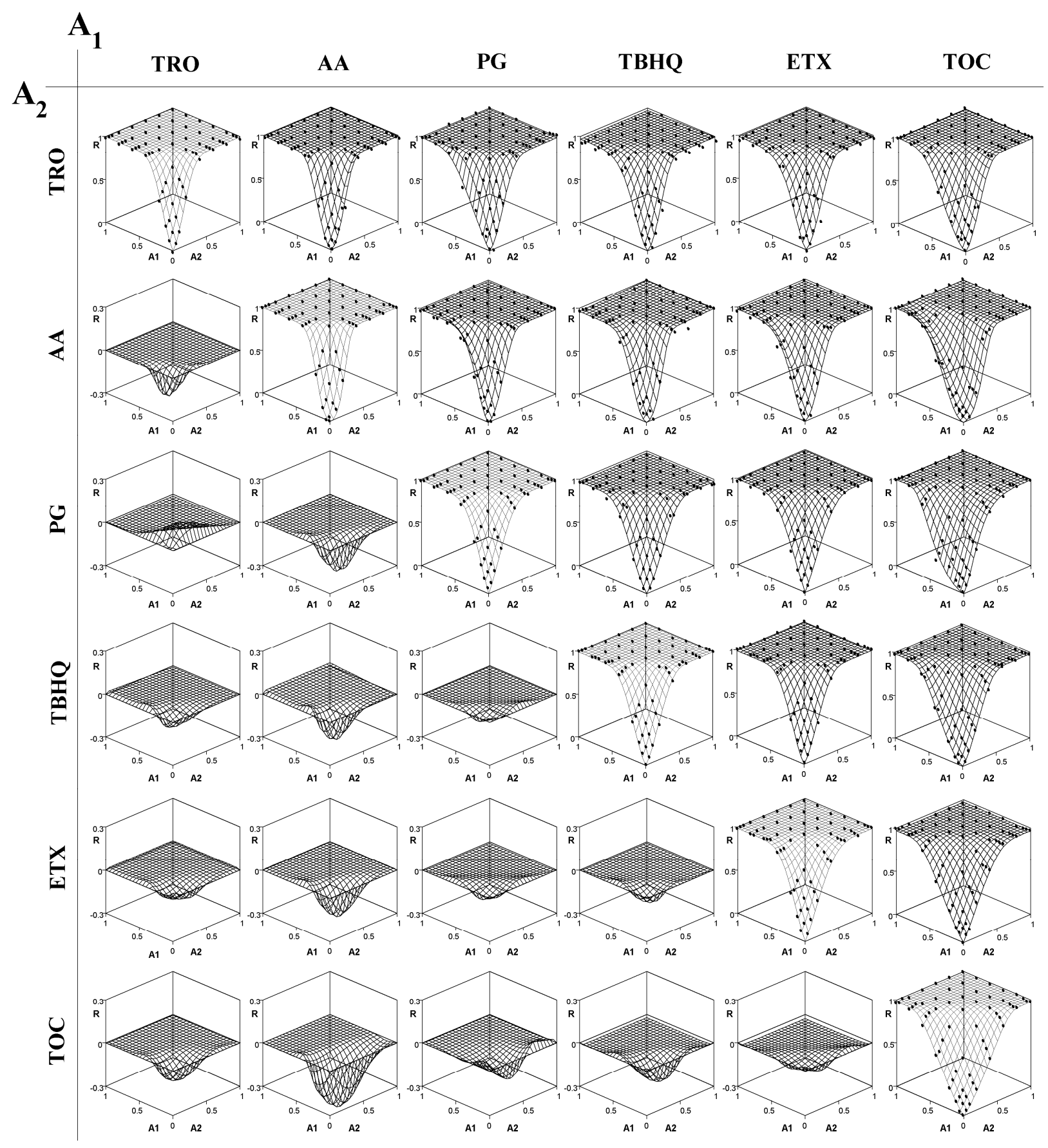

Figure 3: Matrix combination responses for the DPPH assay, which is organized as follows: a) the results obtained for the controls can be seen in the diagonal; b) in the top part of the diagonal the surface responses for each pair antioxidant combination is presented; and c) in the bottom diagonal part, the different "scenery" between their respective null interaction form and the obtained response is presented. Numerical results are in Table 2 and Table A1. 


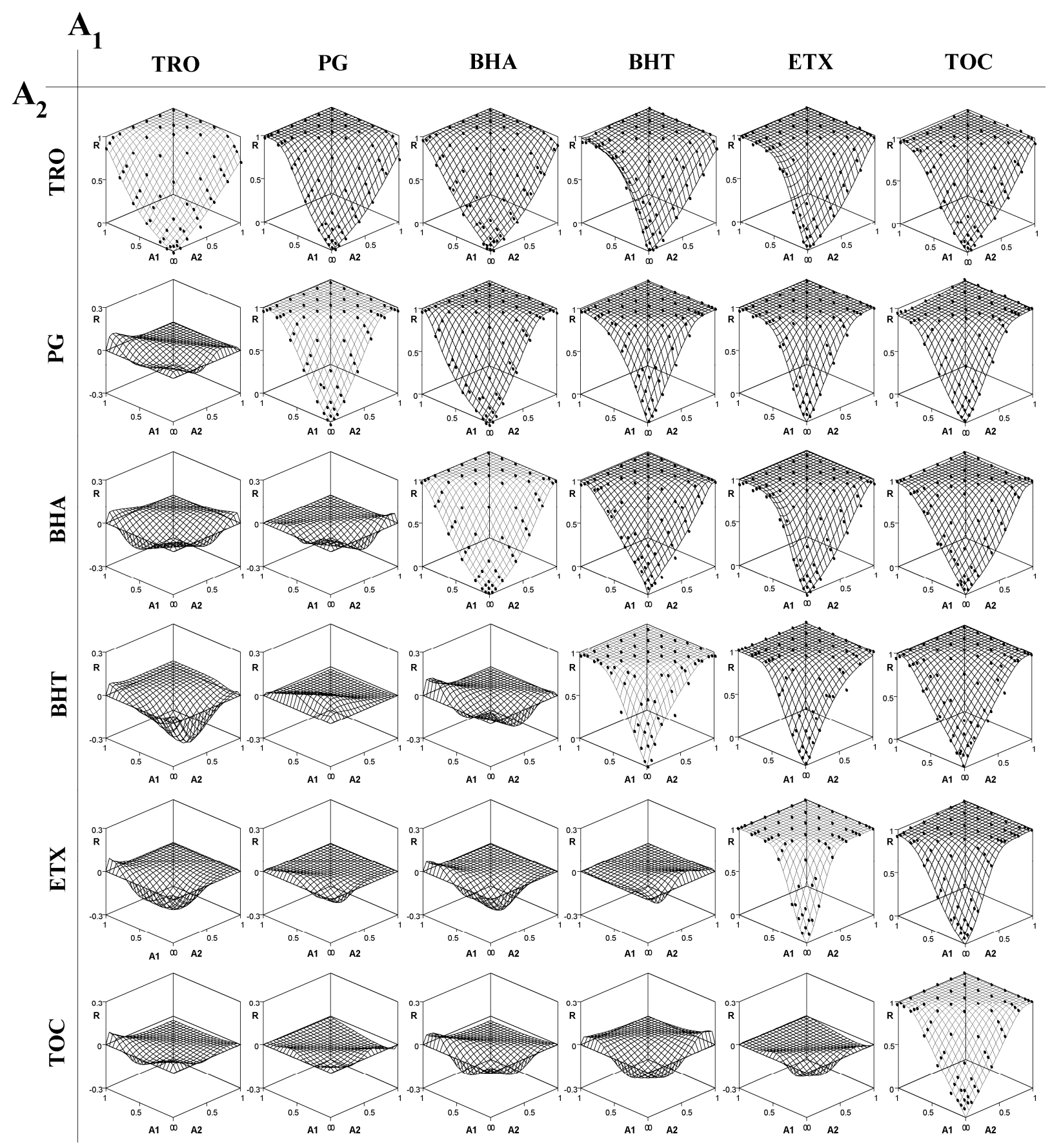

Figure 4: Matrix combination responses for the ABTS assay, which is organized as follows: a) in the diagonal, the results obtained for the controls it can be seen; b) in the top part of the diagonal, the surface responses for each pair antioxidant combination is presented; and c) in the bottom diagonal part, the different "scenery" between their respective null interaction form and the obtained response is presented. Numerical results are in Table 2 and Table A2. 

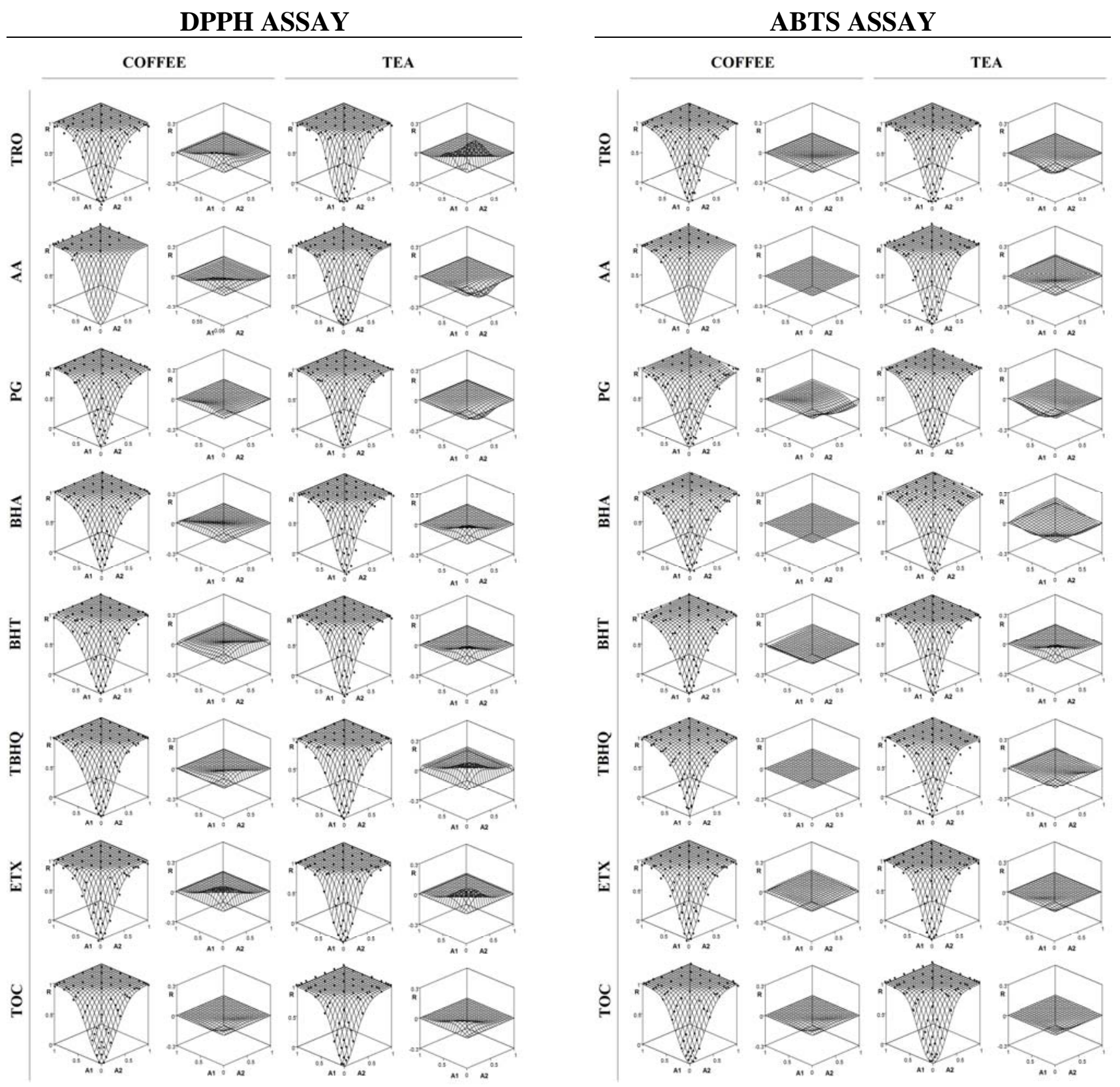

Figure 5: Combination responses for the antioxidant extracts against standard antioxidants in the DPPH and ABTS reactions. To surfaces response graphs for each case for each reaction are show: one belongs to results obtained for the joint response and the other to the differences "scenery" between their respective null interaction form and the obtained response. Numerical results are in Table 2 and Table A3. 


\section{A: RUV REPRODUCIBILITY FOR DPPH ASSAY}

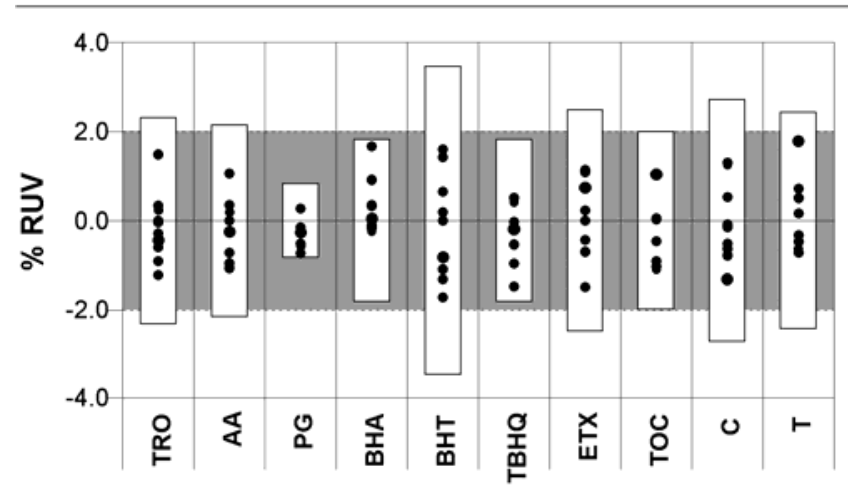

B: RUV REPRODUCIBILITY FOR ABTS ASSAY

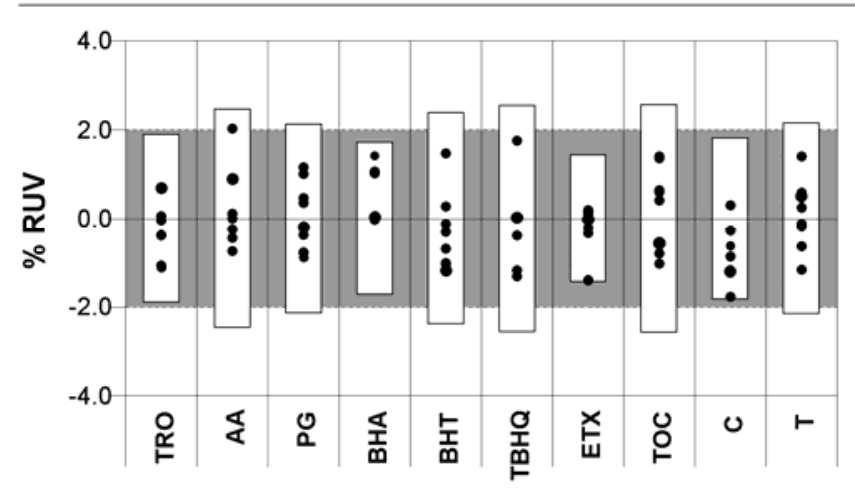

\section{C: TROLOX REPRODUCIBILITY ILLUSTRATION}

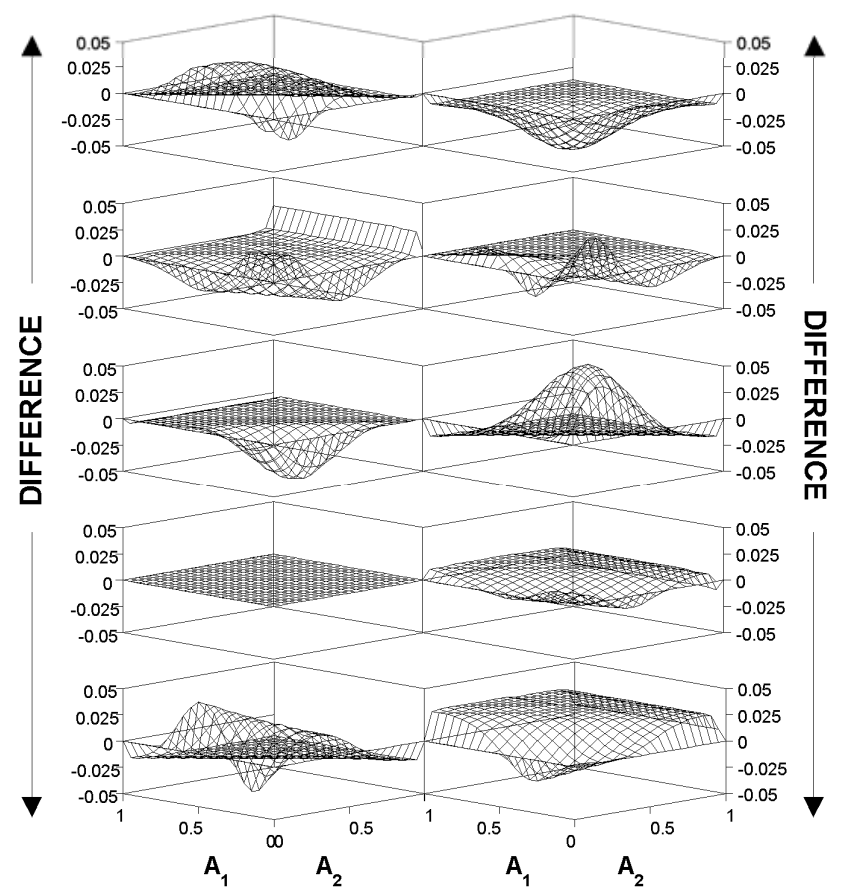

Figure 6: Reproducibility of the methodological procedure and limit of confidence for the \% $R U V$ results. Note that the range of axis $\mathrm{z}$ is six times lower than those presented in Figure 3, Figure 4 and Figure 5. 


\section{TABLES}

Table 1: Parametric estimations and statistic information of the kinetic series of the dose-responses of all antioxidant standards and extracts fitted to the kinetic Eq. (2), (3) and (4).

\begin{tabular}{|c|c|c|c|c|c|c|}
\hline \multirow{2}{*}{ antioxidants } & \multicolumn{3}{|c|}{ dose-effect parameters from Eq. (2) } & \multirow{2}{*}{$\begin{array}{l}\text { statistics } \\
R_{a d j}^{2}\end{array}$} & \multicolumn{2}{|c|}{ additional parameters } \\
\hline & $K$ & $\tau$ & $\alpha$ & & $\boldsymbol{v}_{\tau}(E q .(3))$ & $\boldsymbol{n}_{95 \%}(E q .(4))$ \\
\hline \multicolumn{7}{|l|}{ DPPH ASSAY } \\
\hline$T R O$ & $29.92 \pm 2.4$ & $0.663 \pm 7.6$ & $1.92 \pm 9.6$ & 0.9997 & $30.39 \pm 9.1$ & 1.16 \\
\hline$A A$ & $29.64 \pm 1.6$ & $0.806 \pm 19.2$ & $3.14 \pm 7.6$ & 0.9997 & $40.25 \pm 7.4$ & 1.41 \\
\hline$P G$ & $29.83 \pm 1.7$ & $0.254 \pm 7.2$ & $1.48 \pm 5.5$ & 0.9991 & $60.81 \pm 5.3$ & 0.45 \\
\hline BHA & $27.92 \pm 3.2$ & $0.572 \pm 8.8$ & $1.18 \pm 14.1$ & 0.9985 & $20.37 \pm 12.3$ & 1.00 \\
\hline BHT & $30.10 \pm 1.7$ & $3.614 \pm 7.5$ & $1.39 \pm 5.1$ & 0.9985 & $4.65 \pm 5.0$ & 6.32 \\
\hline TBHQ & $29.96 \pm 1.7$ & $0.468 \pm 5.4$ & $1.80 \pm 6.0$ & 0.9998 & $40.41 \pm 5.8$ & 0.82 \\
\hline ETX & $29.68 \pm 1.0$ & $0.870 \pm 0.3$ & $1.69 \pm 2.5$ & 0.9995 & $20.70 \pm 2.5$ & 1.52 \\
\hline TOC & $29.64 \pm 3.3$ & $0.722 \pm 2.1$ & $1.41 \pm 8.5$ & 0.9994 & $20.31 \pm 8.7$ & 1.26 \\
\hline$T$ & $30.00 \pm 1.5$ & $0.713 \pm 5.4$ & $2.11 \pm 4.9$ & 0.9997 & $30.77 \pm 4.5$ & 1.25 \\
\hline C & $30.00 \pm 2.8$ & $1.829 \pm 0.8$ & $1.51 \pm 8.5$ & 0.9995 & $8.59 \pm 8.2$ & 3.20 \\
\hline \multicolumn{7}{|l|}{ ABTS ASSAY } \\
\hline TRO & $14.73 \pm 3.8$ & $0.158 \pm 1.0$ & $1.39 \pm 7.5$ & 0.9988 & $45.02 \pm 9.6$ & 0.28 \\
\hline$A A$ & $14.53 \pm 2.8$ & $0.580 \pm 2.2$ & $1.66 \pm 6.0$ & 0.9997 & $14.38 \pm 6.6$ & 1.02 \\
\hline$P G$ & $15.93 \pm 3.3$ & $0.097 \pm 1.1$ & $1.63 \pm 5.1$ & 0.9993 & $93.26 \pm 5.7$ & 0.17 \\
\hline BHA & $15.00 \pm 9.1$ & $0.124 \pm 0.8$ & $1.26 \pm 9.2$ & 0.9994 & $52.74 \pm 11.7$ & 0.22 \\
\hline BHT & $15.02 \pm 6.5$ & $0.481 \pm 0.5$ & $0.92 \pm 6.8$ & 0.9994 & $10.01 \pm 14.4$ & 0.84 \\
\hline TBHQ & $15.00 \pm 3.2$ & $0.050 \pm 2.5$ & $0.87 \pm 8.2$ & 0.9992 & $90.62 \pm 9.1$ & 0.09 \\
\hline$E T X$ & $15.16 \pm 2.2$ & $0.157 \pm 9.1$ & $1.73 \pm 6.7$ & 0.9996 & $58.06 \pm 6.8$ & 0.27 \\
\hline TOC & $15.01 \pm 1.8$ & $0.688 \pm 8.0$ & $2.67 \pm 3.1$ & 0.9999 & $20.21 \pm 5.7$ & 1.20 \\
\hline$T$ & $15.03 \pm 0.9$ & $0.246 \pm 5.0$ & $2.42 \pm 2.6$ & 0.9998 & $51.29 \pm 2.8$ & 0.43 \\
\hline$C$ & $14.98 \pm 2.2$ & $0.451 \pm 8.2$ & $1.58 \pm 6.8$ & 0.9994 & $18.25 \pm 6.8$ & 0.79 \\
\hline
\end{tabular}

$\boldsymbol{K}$ ( $\mathrm{nM}$ radical reduced); $\boldsymbol{\tau}$ ( $\mu \mathrm{g}$ of $\mathrm{A}$ ); $\boldsymbol{v}_{\tau}$ (averaged molecules of the reduced radical $\mu \mathrm{\mu}$ of $\left.\mathrm{A}\right) \boldsymbol{n}_{\mathbf{9 5} \%}(\mu \mathrm{g}$ of $\mathrm{A}$ needed to reach the $95 \%$ of the $R$ ). Confidence intervals are showed in \% of the parameter $(\alpha=0.05) . R_{a d j}^{2}:$ correlation coefficient adjusted between observed and predicted values. 
Table 2: Effect of the combination of 45 different pairs of standard antioxidants and extracts for each SET reaction. For the cases that each sample is combined with itself, the results are used simply as a control. For each statistically consistent case the resulting $R U V$ values are presented (ns if $R U V<2.0$ $\%$, see text for more details). The concentration ranges used for each case are showed in $\mu \mathrm{g}$ in for the final reaction volume of the reaction $(300 \mu \mathrm{L})$.

\begin{tabular}{|c|c|c|c|c|c|c|c|c|c|c|c|}
\hline \multicolumn{12}{|c|}{ A: DPPH REACTION } \\
\hline & & TRO & $A \boldsymbol{A}$ & $P G$ & $\boldsymbol{B H} \boldsymbol{A}$ & $B H T$ & TBHQ & $E T X$ & TOC & $T$ & $C$ \\
\hline $0.00-1.25 \mu g$ & TRO & $N I$ & ns & ns & ns & ns & ns & ns & ns & ns & 2.84 \\
\hline $0.00-1.50 \mu g$ & $\boldsymbol{A} \boldsymbol{A}$ & -- & $N I$ & ns & ns & ns & -4.94 & ns & -2.37 & ns & ns \\
\hline $0.00-0.50 \mu \mathrm{g}$ & $P G$ & -- & -- & $N I$ & ns & ns & ns & ns & ns & ns & ns \\
\hline $0.00-1.00 \mu g$ & BHA & -- & -- & -- & $N I$ & ns & ns & -3.21 & -2.31 & ns & ns \\
\hline $0.00-6.25 \mu g$ & BHT & -- & -- & -- & -- & $N I$ & ns & ns & ns & ns & 4.17 \\
\hline $0.00-1.00 \mu g$ & TBHQ & -- & -- & -- & -- & -- & $N I$ & ns & ns & 6.01 & ns \\
\hline $0.00-1.50 \mu \mathrm{g}$ & $E T X$ & -- & -- & -- & -- & -- & -- & $N I$ & ns & 2.79 & 3.16 \\
\hline $0.00-1.25 \mu \mathrm{g}$ & TOC & -- & -- & -- & -- & -- & -- & -- & $N I$ & ns & ns \\
\hline $0.00-1.25 \mu \mathrm{g}$ & $T$ & -- & -- & -- & -- & -- & -- & -- & -- & $N I$ & ns \\
\hline $0.00-3.25 \mu \mathrm{g}$ & $C$ & -- & -- & -- & -- & -- & -- & -- & -- & -- & $N I$ \\
\hline \multicolumn{12}{|c|}{ A: ABTS REACTION } \\
\hline & & TRO & $\boldsymbol{A} \boldsymbol{A}$ & $P G$ & $\boldsymbol{B H} \boldsymbol{A}$ & BHT & $T B H Q$ & ETX & TOC & $T$ & $C$ \\
\hline $0.00-0.30 \mu \mathrm{g}$ & TRO & $N I$ & -7.57 & ns & -2.17 & ns & ns & -2.76 & ns & ns & ns \\
\hline $0.00-1.00 \mu \mathrm{g}$ & $\boldsymbol{A A}$ & -- & $N I$ & -8.10 & -10.16 & ns & -19.55 & -9.52 & -6.00 & ns & ns \\
\hline $0.00-0.20 \mu \mathrm{g}$ & $P G$ & -- & -- & $N I$ & ns & ns & ns & ns & ns & -3.27 & ns \\
\hline $0.00-0.25 \mu \mathrm{g}$ & BHA & -- & -- & -- & $N I$ & ns & ns & -2.35 & -2.27 & ns & ns \\
\hline $0.00-1.00 \mu \mathrm{g}$ & BHT & -- & -- & -- & -- & $N I$ & 3.26 & ns & ns & 2.05 & ns \\
\hline $0.00-0.10 \mu \mathrm{g}$ & TBHQ & -- & -- & -- & -- & -- & $N I$ & ns & 2.29 & ns & ns \\
\hline $0.00-0.30 \mu \mathrm{g}$ & $E T X$ & -- & -- & -- & -- & -- & -- & $N I$ & ns & ns & ns \\
\hline $0.00-1.25 \mu \mathrm{g}$ & TOC & -- & -- & -- & -- & -- & -- & -- & $N I$ & ns & ns \\
\hline $0.00-0.50 \mu \mathrm{g}$ & $T$ & -- & -- & -- & -- & -- & -- & -- & -- & $N I$ & ns \\
\hline $0.00-1.00 \mu g$ & $C$ & -- & -- & -- & -- & -- & -- & -- & -- & -- & $N I$ \\
\hline
\end{tabular}

$N I$ : Null interaction, ns: non statistically significant $R U V$ result. 
Table A1: Parametric values of the joint action between different standard antioxidants in the DPPH reaction by fitting the experimental results to the Eq. (6) (assuming no interactions) and (8) (assuming interactive mechanisms). The degree of interactive effect (\% RUV) is computed as described in Eq. (9). For all the presented parameters its estimations are significant.

\begin{tabular}{|c|c|c|c|c|c|c|c|c|c|c|c|c|c|c|c|c|c|c|c|c|c|c|c|c|}
\hline \multirow{2}{*}{\multicolumn{2}{|c|}{$\begin{array}{l}\text { Antiox. } \\
\text { combiantion }\end{array}$}} & \multicolumn{5}{|c|}{ ASSUMING NO INTERACTIONS } & \multicolumn{17}{|c|}{ ASSUMING INTERACTIONS } & \multirow{3}{*}{$\begin{array}{c}\text { degree o } \\
\text { inter. } \\
\% R U V\end{array}$} \\
\hline & & \multicolumn{3}{|c|}{ joint response } & \multicolumn{2}{|c|}{$\begin{array}{l}\text { relative fitting } \\
\text { potency analysis }\end{array}$} & \multicolumn{3}{|c|}{ joint response } & \multirow{2}{*}{$\begin{array}{c}\text { relative } \\
\text { potency } \\
p\end{array}$} & \multicolumn{2}{|c|}{$\begin{array}{l}\text { A1 altering } \\
\text { eff. conc. of } \\
\text { A2 }\end{array}$} & \multicolumn{2}{|c|}{$\begin{array}{l}\text { A2 altering } \\
\text { eff. conc. of } \\
\text { A1 }\end{array}$} & \multicolumn{4}{|c|}{$\begin{array}{c}\text { A1 as perturbing factor } \\
\text { for params. of the joint } \\
\text { response }\end{array}$} & \multicolumn{4}{|c|}{$\begin{array}{l}\text { A2 as perturbing factor } \\
\text { for params. of the joint } \\
\text { response }\end{array}$} & \multirow{2}{*}{$\begin{array}{c}\text { fitting } \\
\text { analysis } \\
R_{a d j}^{2}\end{array}$} & \\
\hline A1 & A2 & $K$ & $m$ & $\alpha$ & $p$ & $R_{a d j}^{2}$ & $K$ & $m$ & $\alpha$ & & $b_{D 2}$ & $c_{D 2}$ & $b_{D l}$ & $c_{D I}$ & $b_{k 2}$ & $c_{k 2}$ & $b_{m 2}$ & $c_{m 2}$ & $b_{k l}$ & $c_{k l}$ & $b_{m l}$ & $c_{m l}$ & & \\
\hline$T R O$ & TRO & 1.000 & 0.176 & 1.721 & 0.983 & 0.9892 & 1.000 & 0.176 & 1.721 & 0.983 & -- & -- & -- & -- & -- & -- & -- & -- & -- & -- & -- & -- & 0.9892 & 0.00 \\
\hline TRO & $A A$ & 0.999 & 0.206 & 2.058 & 1.012 & 0.9793 & 0.992 & 0.272 & 1.703 & 0.795 & -- & -- & -- & -- & -- & -- & 3.106 & -- & -- & -- & 9.648 & -- & 0.9957 & -1.43 \\
\hline$T R O$ & $P G$ & 0.999 & 0.293 & 1.572 & 1.492 & 0.9756 & 0.985 & 0.395 & 1.155 & 0.535 & -- & -- & -- & -- & -- & -- & 11.209 & -- & -- & -- & -- & -- & 0.9896 & 0.33 \\
\hline$T R O$ & BHA & 0.938 & 0.102 & 1.628 & 0.541 & 0.8951 & 0.899 & 0.102 & 1.257 & 0.423 & -- & -- & -- & 0.129 & -- & -0.990 & -- & -- & -- & -- & 29.614 & -- & 0.9802 & 0.49 \\
\hline$T R O$ & $B H T$ & 0.979 & 0.229 & 1.813 & 1.157 & 0.9886 & 0.994 & 0.233 & 1.694 & 0.888 & -- & -- & -- & -- & -- & -0.990 & -- & -- & -- & -- & 2.487 & -0.024 & 0.9965 & -0.41 \\
\hline$T R O$ & $T B H Q$ & 0.980 & 0.215 & 1.953 & 0.958 & 0.9851 & 0.987 & 0.216 & 1.589 & 0.721 & -- & -0.889 & -- & -- & -- & -0.990 & -- & -- & -- & -- & -- & -- & 0.9925 & 0.09 \\
\hline$T R O$ & ETX & 1.000 & 0.333 & 1.765 & 1.652 & 0.9800 & 0.994 & 0.451 & 1.509 & 1.680 & -- & -- & -- & -- & -- & -0.990 & -- & -- & -- & -- & 2.073 & -- & 0.9938 & -1.71 \\
\hline$T R O$ & TOC & 1.000 & 0.203 & 2.589 & 0.928 & 0.9916 & 0.997 & 0.208 & 2.571 & 0.747 & -- & -- & -- & -- & -- & -0.990 & -- & -- & -- & -- & 3.715 & -- & 0.9980 & -0.74 \\
\hline$A A$ & $A A$ & 0.984 & 0.201 & 1.935 & 0.926 & 0.9848 & 0.984 & 0.201 & 1.935 & 0.926 & -- & -- & -- & -- & -- & -- & -- & -- & -- & -- & -- & -- & 0.9848 & 0.00 \\
\hline$A A$ & $P G$ & 0.951 & 0.115 & 1.959 & 0.529 & 0.9181 & 0.991 & 0.101 & 1.650 & 0.318 & -- & -- & 4.334 & -- & -- & -0.990 & -- & -- & -- & -- & -- & -- & 0.9893 & -0.39 \\
\hline$A A$ & BHA & 0.973 & 0.239 & 2.205 & 1.074 & 0.9742 & 0.962 & 0.238 & 2.237 & 0.809 & -- & -- & 4.381 & -- & -0.032 & -0.990 & -- & -- & -- & -- & -- & -- & 0.9938 & -0.63 \\
\hline$A A$ & $B H T$ & 0.973 & 0.227 & 1.864 & 1.019 & 0.9658 & 0.975 & 0.226 & 1.704 & 0.745 & -- & -- & 7.994 & -- & -- & -0.990 & -- & -- & -- & -- & -0.990 & -- & 0.9911 & -1.27 \\
\hline$A A$ & $T B H Q$ & 0.999 & 0.333 & 2.185 & 1.321 & 0.9224 & 0.998 & 0.330 & 2.194 & 0.810 & -- & -- & 6.337 & -- & -- & -0.990 & -- & -- & -- & -- & -- & -- & 0.9835 & -4.94 \\
\hline$A A$ & ETX & 1.000 & 0.164 & 1.349 & 0.848 & 0.9849 & 0.991 & 0.209 & 1.182 & 0.834 & -- & -- & 2.802 & -- & -- & -0.990 & -- & -- & -- & -- & -- & -- & 0.9958 & -1.01 \\
\hline$A A$ & TOC & 0.970 & 0.106 & 1.281 & 0.544 & 0.9576 & 0.969 & 0.104 & 1.098 & 0.387 & -- & -- & 2.280 & -- & -- & -0.990 & -- & -- & -- & -- & 5.605 & -- & 0.9913 & -2.37 \\
\hline$P G$ & $P G$ & 0.981 & 0.253 & 1.675 & 1.074 & 0.9910 & 0.981 & 0.253 & 1.675 & 1.074 & -- & -- & -- & -- & -- & -- & -- & -- & -- & -- & -- & -- & 0.9910 & 0.00 \\
\hline$P G$ & BHA & 1.000 & 0.209 & 1.368 & 1.009 & 0.9870 & 0.989 & 0.279 & 1.105 & 0.942 & -- & -- & -- & -- & -- & -0.990 & -- & -- & -- & -- & -- & -- & 0.9939 & -0.66 \\
\hline$P G$ & $B H T$ & 1.000 & 0.410 & 1.795 & 1.828 & 0.9546 & 0.996 & 0.902 & 1.477 & 4.531 & -- & -- & -- & -- & -- & -- & -- & -- & -- & -0.990 & -- & -- & 0.9766 & -1.83 \\
\hline$P G$ & $T B H Q$ & 0.983 & 0.101 & 1.120 & 0.890 & 0.9792 & 0.979 & 0.100 & 1.252 & 1.016 & -- & -- & 3.134 & -- & -- & 0.940 & -- & -- & -- & -- & -- & -- & 0.9876 & $\begin{array}{l}-3.29 \\
\end{array}$ \\
\hline$P G$ & ETX & 0.945 & 0.214 & 1.508 & 1.952 & 0.9507 & 0.983 & 0.307 & 1.378 & 3.577 & -- & -- & -- & -- & -- & 2.607 & -- & -- & -- & -- & 2.110 & -- & 0.9917 & -0.78 \\
\hline$P G$ & TOC & 0.957 & 0.181 & 1.381 & 1.616 & 0.9664 & 0.975 & 0.227 & 1.322 & 2.555 & -- & -- & 11.276 & -- & -- & 2.092 & -- & -- & -- & -- & -- & -- & 0.9928 & -0.42 \\
\hline$B H A$ & $B H A$ & 0.944 & 0.291 & 1.457 & 2.521 & 0.9814 & 0.944 & 0.291 & 1.457 & 2.521 & -- & -- & -- & -- & -- & -- & -- & -- & -- & -- & -- & -- & 0.9814 & 0.00 \\
\hline$B H A$ & $B H T$ & 1.000 & 0.200 & 1.624 & 0.905 & 0.9886 & 0.990 & 0.264 & 1.323 & 0.930 & -- & -- & 1.955 & -- & -- & -0.990 & -- & -- & -- & -- & -- & --- & 0.9956 & -1.19 \\
\hline BHA & $T B H Q$ & 1.000 & 0.200 & 1.624 & 0.905 & 0.9886 & 0.990 & 0.264 & 1.323 & 0.930 & -- & -- & 1.955 & -- & -- & -0.990 & -- & -- & -- & -- & -- & -- & 0.9956 & -1.19 \\
\hline BHA & ETX & 1.000 & 0.337 & 1.770 & 1.343 & 0.9789 & 0.978 & 0.542 & 1.361 & 1.685 & -- & -- & 4.429 & -- & -- & -0.990 & -- & -- & -- & -- & -- & -- & 0.9894 & -3.21 \\
\hline$B H A$ & TOC & 1.000 & 0.187 & 1.478 & 0.826 & 0.9868 & 0.985 & 0.237 & 1.203 & 0.839 & -- & -- & 3.456 & -- & -- & -0.767 & -- & -- & -- & -- & -- & -- & 0.9960 & -2.31 \\
\hline$B H T$ & $B H T$ & 1.000 & 0.310 & 1.510 & 1.211 & 0.9888 & 1.000 & 0.310 & 1.510 & 0.9888 & -- & -- & -- & -- & -- & -- & -- & -- & -- & -- & -- & -- & 0.9932 & 0.00 \\
\hline BHT & $T B H Q$ & 1.000 & 0.349 & 1.690 & 0.973 & 0.9933 & 0.979 & 0.577 & 1.133 & 0.947 & -- & -- & -0.740 & -- & -- & -0.990 & -- & -- & -- & -- & -- & -- & 0.9981 & -0.58 \\
\hline$B H T$ & ETX & 1.000 & 0.176 & 1.721 & 0.983 & 0.9892 & 0.998 & 0.219 & 1.394 & 0.854 & -- & -- & 5.176 & -- & -- & -- & 2.397 & -- & -- & -- & -- & -- & 0.9981 & -0.69 \\
\hline$B H T$ & TOC & 0.999 & 0.206 & 2.058 & 1.012 & 0.9793 & 0.992 & 0.272 & 1.703 & 0.795 & -- & -- & -- & -- & -- & -- & 3.106 & -- & -- & -- & 9.648 & -- & 0.9957 & -1.43 \\
\hline$T B H Q$ & $T B H Q$ & 0.999 & 0.293 & 1.572 & 1.492 & 0.9756 & 0.999 & 0.293 & 1.572 & 1.492 & -- & -- & -- & -- & -- & -- & -- & -- & -- & -- & -- & -- & 0.9756 & 0.00 \\
\hline$T B H Q$ & ETX & 0.938 & 0.102 & 1.628 & 0.541 & 0.8951 & 0.899 & 0.102 & 1.257 & 0.423 & -- & -- & -- & 0.129 & -- & -0.990 & -- & -- & -- & -- & 29.614 & -- & 0.9802 & 0.49 \\
\hline$T B H Q$ & $T O C$ & 0.979 & 0.229 & 1.813 & 1.157 & 0.9886 & 0.994 & 0.233 & 1.694 & 0.888 & -- & -- & -- & -- & -- & -0.990 & -- & -- & -- & -- & 2.487 & -0.024 & 0.9965 & -0.41 \\
\hline ETX & ETX & 0.980 & 0.215 & 1.953 & 0.958 & 0.9851 & 0.980 & 0.215 & 1.953 & 0.958 & -- & -- & -- & -- & -- & -- & -- & -- & -- & -- & -- & -- & 0.9925 & 0.00 \\
\hline ETX & TOC & 1.000 & 0.333 & 1.765 & 1.652 & 0.9800 & 0.994 & 0.451 & 1.509 & 1.680 & -- & -- & -- & -- & -- & -0.990 & -- & -- & -- & -- & 2.073 & -- & 0.9800 & -1.71 \\
\hline TOC & $T O C$ & 1.000 & 0.203 & 2.589 & 0.928 & 0.9916 & 1.000 & 0.203 & 2.589 & 0.928 & -- & -- & -- & -- & -- & -- & -- & -- & -- & -- & -- & -- & 0.9916 & 0.00 \\
\hline
\end{tabular}


Table A2: Parametric values of the joint action between different standard antioxidants in the ABTS reaction by fitting the experimental results to the Eq. (6) (assuming no interactions) and (8) (assuming interactive mechanisms). The degree of interactive effect (\% RUV) is computed as described in Eq. (9). For all the presented parameters its estimations are significant.

\begin{tabular}{|c|c|c|c|c|c|c|c|c|c|c|c|c|c|c|c|c|c|c|c|c|c|c|c|c|}
\hline \multirow{2}{*}{\multicolumn{2}{|c|}{$\begin{array}{l}\text { Antiox. } \\
\text { combiantion }\end{array}$}} & \multicolumn{5}{|c|}{ ASSUMING NO INTERACTIONS } & \multicolumn{17}{|c|}{ ASSUMING INTERACTIONS } & \multirow{3}{*}{$\begin{array}{c}\text { degree of } \\
\text { inter. } \\
\% R U V\end{array}$} \\
\hline & & \multicolumn{3}{|c|}{ joint response } & \multicolumn{2}{|c|}{$\begin{array}{l}\text { relative fitting } \\
\text { potency analysis }\end{array}$} & \multicolumn{3}{|c|}{ joint response } & \multirow{2}{*}{$\begin{array}{c}\text { relative } \\
\text { potency } \\
p\end{array}$} & \multicolumn{2}{|c|}{$\begin{array}{l}\text { A1 altering } \\
\text { eff. conc. of } \\
\text { A2 }\end{array}$} & \multicolumn{2}{|c|}{$\begin{array}{l}\text { A2 altering } \\
\text { eff. conc. of } \\
\text { A1 }\end{array}$} & \multicolumn{4}{|c|}{$\begin{array}{l}\text { A1 as perturbing factor } \\
\text { for params. of the joint } \\
\text { response }\end{array}$} & \multicolumn{4}{|c|}{$\begin{array}{c}\text { A2 as perturbing factor } \\
\text { for params. of the joint } \\
\text { response }\end{array}$} & \multirow{2}{*}{$\begin{array}{c}\text { fitting } \\
\text { analysis } \\
R_{a d j}^{2}\end{array}$} & \\
\hline A1 & A2 & $K$ & $m$ & $\alpha$ & $p$ & $R_{a d j}^{2}$ & $K$ & $m$ & $\alpha$ & & $b_{D 2}$ & $c_{D 2}$ & $b_{D 1}$ & $c_{D 1}$ & $b_{k 2}$ & $c_{k 2}$ & $b_{m 2}$ & $c_{m 2}$ & $b_{k l}$ & $c_{k 1}$ & $b_{m l}$ & $c_{m l}$ & & \\
\hline$T R O$ & $T R O$ & 1.000 & 0.673 & 2.176 & 0.917 & 0.9876 & 1.000 & 0.673 & 2.176 & 0.917 & -- & -- & -- & -- & -- & -- & -- & -- & -- & -- & -- & -- & 0.9876 & 0.00 \\
\hline$T R O$ & $A A$ & 1.000 & 0.663 & 2.956 & 0.924 & 0.9559 & 1.000 & 2.492 & 1.526 & 1.912 & -- & -- & -- & -- & -- & -0.559 & -- & -- & -- & -- & -- & -- & 0.9791 & -7.57 \\
\hline$T R O$ & $P G$ & 1.000 & 0.404 & 1.908 & 0.583 & 0.9888 & 0.997 & 0.772 & 1.119 & 0.522 & -- & -- & -0.681 & -- & -- & -0.673 & -- & -- & -- & -- & -- & -- & 0.9965 & 1.56 \\
\hline$T R O$ & $B H A$ & 1.000 & 0.519 & 1.833 & 0.862 & 0.9703 & 0.999 & 1.105 & 0.982 & 0.677 & -- & -- & -- & -- & -- & -0.814 & -- & -- & -- & -- & -0.765 & -- & 0.9900 & -2.17 \\
\hline$T R O$ & BHT & 0.955 & 0.216 & 1.890 & 0.353 & 0.9464 & 0.996 & 0.225 & 1.273 & 0.218 & -- & -- & 6.844 & -- & -- & -0.549 & -- & -- & -- & 6.017 & -- & -- & 0.9878 & -1.98 \\
\hline$T R O$ & $T B H Q$ & 1.000 & 0.622 & 1.971 & 1.052 & 0.9852 & 1.000 & 1.366 & 1.028 & 0.616 & -- & -- & -- & -- & -- & -0.708 & 1.454 & -- & -- & -- & -- & -- & 0.9963 & -0.44 \\
\hline$T R O$ & ETX & 0.988 & 0.231 & 1.798 & 0.411 & 0.9669 & 0.995 & 0.630 & 1.024 & 0.450 & -- & -- & 3.303 & -- & -- & -0.841 & -- & -- & -- & -- & -- & -- & 0.9919 & -2.76 \\
\hline$T R O$ & $T O C$ & 1.000 & 0.439 & 1.884 & 0.759 & 0.9837 & 0.971 & 1.942 & 0.844 & 1.307 & -- & -- & -- & -- & -- & -0.914 & -- & -- & -- & -- & -- & -- & 0.9923 & -1.31 \\
\hline$A A$ & $A A$ & 1.000 & 0.652 & 7.156 & 1.047 & 0.9810 & 1.000 & 0.652 & 7.156 & 1.047 & -- & -- & -- & -- & -- & -- & -- & -- & -- & -- & -- & -- & 0.9810 & 0.00 \\
\hline$A A$ & $P G$ & 1.000 & 0.429 & 3.207 & 0.712 & 0.9426 & 1.000 & 0.477 & 1.687 & 0.254 & -- & -- & 1.988 & -- & -- & -0.990 & -- & -- & -- & -- & -- & -- & 0.9967 & -8.10 \\
\hline$A A$ & $B H A$ & 1.000 & 0.601 & 2.997 & 0.870 & 0.9356 & 0.999 & 1.008 & 1.510 & 0.456 & -- & -- & 1.792 & -- & -- & -0.990 & -- & -- & -- & -- & -- & -- & 0.9868 & -10.16 \\
\hline$A A$ & BHT & 1.000 & 0.363 & 1.364 & 0.407 & 0.8657 & 1.000 & 0.328 & 1.162 & 0.191 & -- & -- & 1.392 & -- & -- & -0.781 & -- & -- & -- & -- & -- & -- & 0.9233 & 0.81 \\
\hline$A A$ & $T B H Q$ & 1.000 & 0.643 & 2.939 & 0.935 & 0.8978 & 1.000 & 1.215 & 1.188 & 0.400 & -- & -0.990 & -- & -- & -- & -0.990 & -- & -- & -- & -- & -- & -- & 0.9897 & -19.55 \\
\hline$A A$ & ETX & 0.982 & 0.287 & 2.956 & 0.420 & 0.9068 & 0.982 & 0.387 & 1.275 & 0.124 & -- & -- & 2.711 & -- & -- & -0.990 & -- & -- & -- & -- & -- & -- & 0.9899 & -9.52 \\
\hline$A A$ & $T O C$ & 0.961 & 0.424 & 2.463 & 0.649 & 0.9283 & 1.000 & 0.396 & 1.467 & 0.145 & -- & -- & 1.515 & -- & -- & -0.990 & -- & -- & -- & -- & -- & -- & 0.9866 & -6.00 \\
\hline$P G$ & $P G$ & 1.000 & 0.384 & 1.876 & 0.999 & 0.9935 & 1.000 & 0.384 & 1.876 & 0.999 & -- & -- & -- & -- & -- & -- & -- & -- & -- & -- & -- & -- & 0.9935 & 0.00 \\
\hline$P G$ & $B H A$ & 1.000 & 0.559 & 2.170 & 1.412 & 0.9765 & 0.999 & 1.510 & 1.121 & 2.202 & -- & -- & -0.990 & -- & -- & -0.990 & -- & -- & -- & -- & -- & -- & 0.9886 & -0.83 \\
\hline$P G$ & BHT & 1.000 & 0.267 & 1.406 & 0.816 & 0.9800 & 0.983 & 0.312 & 1.048 & 0.554 & -- & 3.272 & -- & -- & -- & -0.990 & -- & -- & -- & -- & -- & -- & 0.9944 & 1.65 \\
\hline$P G$ & $T B H Q$ & 1.000 & 0.598 & 1.681 & 1.775 & 0.9822 & 0.991 & 1.220 & 1.016 & 2.209 & -- & 2.340 & -- & -- & -- & -0.990 & -- & -- & -- & -- & -- & -- & 0.9890 & 0.94 \\
\hline$P G$ & ETX & 1.000 & 0.245 & 1.562 & 0.769 & 0.9850 & 0.996 & 0.643 & 1.118 & 1.302 & -- & -- & 3.917 & -- & -- & -0.866 & -- & -- & -- & -- & -- & -- & 0.9944 & -0.22 \\
\hline$P G$ & TOC & 1.000 & 0.432 & 1.695 & 1.296 & 0.9929 & 0.916 & 0.684 & 1.159 & 1.461 & -- & -- & -- & 0.084 & -- & -0.969 & -- & -- & -- & -- & -- & -- & 0.9974 & -1.70 \\
\hline$B H A$ & $B H A$ & 1.000 & 0.559 & 2.283 & 0.995 & 0.9921 & 1.000 & 0.559 & 2.283 & 0.995 & -- & -- & -- & -- & -- & -- & -- & -- & -- & -- & -- & -- & 0.9921 & 0.00 \\
\hline BHA & BHT & 1.000 & 0.321 & 1.420 & 0.668 & 0.9433 & 0.987 & 0.632 & 0.716 & 0.556 & -- & -- & -0.990 & -- & -- & -0.990 & -- & -- & -- & -- & -- & -- & 0.9825 & -0.65 \\
\hline$B H A$ & $T B H Q$ & 1.000 & 0.625 & 1.892 & 1.229 & 0.9839 & 1.000 & 1.461 & 0.990 & 1.311 & -- & -- & -- & -- & -- & -0.881 & -- & -- & -- & -- & -0.731 & -- & 0.9962 & 0.24 \\
\hline$B H A$ & ETX & 1.000 & 0.250 & 1.765 & 0.494 & 0.9747 & 0.994 & 0.847 & 1.117 & 0.798 & -- & -- & 5.543 & -- & -- & -0.830 & -- & -- & -- & -- & -- & -- & 0.9946 & -2.35 \\
\hline BHA & TOC & 1.000 & 0.356 & 1.506 & 0.764 & 0.9620 & 0.968 & 0.639 & 0.961 & 0.536 & -- & -- & -0.685 & -- & -- & -0.990 & -- & -- & -- & -- & -- & -- & 0.9844 & -2.27 \\
\hline$B H T$ & $B H T$ & 1.000 & 0.263 & 1.212 & 0.936 & 0.9832 & 1.000 & 0.263 & 1.212 & 0.936 & -- & -- & -- & -- & -- & -- & -- & -- & -- & -- & -- & -- & 0.9832 & 0.00 \\
\hline$B H T$ & $T B H Q$ & 1.000 & 0.634 & 1.152 & 2.260 & 0.9565 & 0.978 & 1.640 & 0.778 & 3.637 & -- & -- & -- & -- & -- & -0.990 & -- & -- & -- & -- & -0.632 & -- & 0.9821 & 3.26 \\
\hline$B H T$ & ETX & 1.000 & 0.271 & 1.403 & 0.832 & 0.9767 & 0.999 & 0.464 & 1.267 & 1.477 & -- & -- & -- & -- & -- & -- & -- & -- & -- & -- & -- & -- & 0.9884 & 0.66 \\
\hline$B H T$ & TOC & 1.000 & 0.351 & 1.127 & 1.236 & 0.9392 & 0.994 & 0.753 & 0.797 & 1.383 & -- & -- & -- & -- & -- & -0.990 & -- & -- & -- & -- & -- & -- & 0.9720 & 0.11 \\
\hline$T B H Q$ & $T B H Q$ & 1.000 & 0.624 & 2.058 & 0.996 & 0.9847 & 1.000 & 0.624 & 2.058 & 0.996 & -- & -- & -- & -- & -- & -- & -- & -- & -- & -- & -- & -- & 0.9847 & 0.00 \\
\hline$T B H Q$ & ETX & 1.000 & 0.296 & 1.953 & 0.477 & 0.9867 & 0.991 & 0.988 & 1.052 & 0.669 & -- & -- & -- & -- & -- & -0.804 & -- & -- & -- & -- & -- & -- & 0.9969 & -1.01 \\
\hline$T B H Q$ & TOC & 1.000 & 0.416 & 1.844 & 0.711 & 0.9768 & 1.000 & 0.722 & 0.867 & 0.564 & -- & -- & -0.920 & -- & -- & -0.831 & -- & -- & -- & -- & -- & -- & 0.9857 & 2.29 \\
\hline ETX & ETX & 1.000 & 0.220 & 1.561 & 0.990 & 0.9764 & 0.998 & 0.346 & 1.379 & 1.156 & -- & -- & -- & -- & -- & -0.990 & -- & -- & -- & -- & -- & -- & 0.9865 & 0.09 \\
\hline$E T X$ & TOC & 1.000 & 0.413 & 1.592 & 1.632 & 0.9801 & 0.939 & 0.642 & 1.358 & 1.731 & -- & -- & -- & 0.068 & -- & -0.990 & -- & -- & -- & -- & -- & -- & 0.9928 & -1.61 \\
\hline TOC & TOC & 1.000 & 0.389 & 1.572 & 1.007 & 0.9814 & 1.000 & 0.389 & 1.572 & 1.007 & -- & -- & -- & -- & -- & -- & -- & -- & -- & -- & -- & -- & 0.9814 & 0.00 \\
\hline
\end{tabular}


Table A3: Parametric values of the joint action of different standard antioxidants against two natural extracts (T and C) in the DPPH and ABTS reaction by fitting the experimental results to the Eq. (6) (assuming no interactions) and (8) (assuming interactive mechanisms). The degree of interactive effect (\% RUV) is computed as described in Eq. (9). For all the presented parameters its estimations are significant.

\begin{tabular}{|c|c|c|c|c|c|c|c|c|c|c|c|c|c|c|c|c|c|c|c|c|c|c|c|c|}
\hline \multirow{2}{*}{\multicolumn{2}{|c|}{$\begin{array}{c}\text { Antiox. } \\
\text { combiantion }\end{array}$}} & \multicolumn{5}{|c|}{ ASSUMING NO INTERACTIONS } & \multicolumn{17}{|c|}{ ASSUMING INTERACTIONS } & \multirow{3}{*}{$\begin{array}{c}\begin{array}{c}\text { degree of } \\
\text { inter. }\end{array} \\
\% R U V\end{array}$} \\
\hline & & \multicolumn{3}{|c|}{ joint response } & \multirow{2}{*}{$\begin{array}{c}\text { relative } \\
\text { potency } \\
p\end{array}$} & \multirow{2}{*}{$\begin{array}{c}\text { fitting } \\
\text { analysis } \\
R_{\text {adj }}^{2}\end{array}$} & \multicolumn{3}{|c|}{ joint response } & \multirow{2}{*}{$\begin{array}{c}\text { relative } \\
\text { potency } \\
p\end{array}$} & \multicolumn{2}{|c|}{$\begin{array}{l}\text { A1 altering } \\
\text { eff. conc. of } \\
\text { A2 }\end{array}$} & \multicolumn{2}{|c|}{$\begin{array}{l}\text { A2 altering } \\
\text { eff. conc. of } \\
\text { A1 }\end{array}$} & \multicolumn{4}{|c|}{$\begin{array}{l}\text { A1 as perturbing factor } \\
\text { for params. of the joint } \\
\text { response }\end{array}$} & \multicolumn{4}{|c|}{$\begin{array}{l}\text { A2 as perturbing factor } \\
\text { for params. of the joint } \\
\text { response }\end{array}$} & \multirow{2}{*}{$\begin{array}{c}\text { fitting } \\
\text { analysis } \\
R_{a d j}^{2}\end{array}$} & \\
\hline A1 & A2 & $K$ & $m$ & $\alpha$ & & & $K$ & $m$ & $\alpha$ & & $b_{D 2}$ & $c_{D 2}$ & $b_{D 1}$ & $c_{D 1}$ & $b_{k 2}$ & $c_{k 2}$ & $b_{m 2}$ & $c_{m 2}$ & $b_{k l}$ & $c_{k l}$ & $b_{m l}$ & $c_{m l}$ & & \\
\hline \multicolumn{25}{|c|}{ RESPONSES FOR THE DPPH REACTION } \\
\hline TRO & $C$ & 0.978 & 0.303 & 1.833 & 0.959 & 0.9915 & 0.992 & 0.271 & 1.873 & 0.883 & -- & -- & -0.990 & -- & -- & -- & -- & -- & -- & -- & -- & -- & 0.9968 & 2.84 \\
\hline$A A$ & $C$ & 0.999 & 0.361 & 2.267 & 1.009 & 0.9934 & 0.987 & 0.477 & 1.742 & 1.025 & -- & -- & -0.990 & -- & -- & -0.707 & -- & -- & -- & -- & -- & -- & 0.9975 & 0.48 \\
\hline$P G$ & $C$ & 0.995 & 0.279 & 1.566 & 0.990 & 0.9920 & 0.993 & 0.281 & 1.426 & 0.893 & -- & -- & -- & -- & -- & -- & 0.715 & -- & -- & -- & -- & -- & 0.9949 & 0.44 \\
\hline BHA & $C$ & 1.000 & 0.262 & 1.311 & 0.945 & 0.9913 & 0.996 & 0.272 & 1.347 & 1.022 & -- & -- & -0.990 & -- & -- & -- & -- & -- & -- & -- & -- & -- & 0.9953 & 1.84 \\
\hline BHT & $C$ & 0.961 & 0.312 & 1.610 & 0.970 & 0.9864 & 0.985 & 0.333 & 1.461 & 1.002 & -- & -- & -- & -- & -- & -- & -- & -- & -- & -- & -0.990 & -- & 0.9932 & 4.17 \\
\hline$T B H Q$ & $C$ & 1.000 & 0.289 & 1.638 & 0.963 & 0.9886 & 0.993 & 0.325 & 1.587 & 1.010 & -- & -- & -- & -- & -- & -- & -- & -- & -- & -- & -0.954 & -- & 0.9941 & 0.70 \\
\hline ETX & $C$ & 0.985 & 0.292 & 1.676 & 0.981 & 0.9826 & 0.990 & 0.908 & 1.013 & 0.927 & -- & -- & -- & -- & -- & -- & 7.939 & -- & -- & -- & -- & -- & 0.9950 & 3.16 \\
\hline TOC & $C$ & 0.991 & 0.349 & 2.066 & 1.057 & 0.9976 & 0.989 & 0.680 & 1.311 & 0.957 & -- & -- & -- & -- & -- & -- & 3.519 & -- & -- & -- & 2.498 & -- & 0.9978 & 0.11 \\
\hline TRO & $T$ & 1.000 & 0.328 & 2.065 & 1.022 & 0.9686 & 0.990 & 0.313 & 1.753 & 0.098 & -- & -- & 8.059 & -- & -- & -- & 24.824 & -- & -- & -- & -- & -- & 0.9938 & 1.88 \\
\hline$A A$ & $T$ & 0.998 & 0.359 & 2.646 & 1.024 & 0.9954 & 0.991 & 0.353 & 2.465 & 1.032 & -- & -- & 4.841 & -- & -- & -- & -- & -- & -- & 1.854 & -- & -- & 0.9985 & -1.36 \\
\hline$P G$ & $T$ & 0.984 & 0.280 & 1.837 & 1.032 & 0.9947 & 0.989 & 0.282 & 1.776 & 1.127 & -- & -- & 5.208 & -- & -- & -- & -0.210 & -- & -- & 1.792 & -- & -- & 0.9978 & -0.30 \\
\hline BHA & $T$ & 0.991 & 0.259 & 1.741 & 0.988 & 0.9904 & 0.994 & 0.248 & 1.724 & 0.950 & -- & -- & -- & -- & -- & -- & -- & -- & -- & 2.046 & -- & -- & 0.9964 & 1.64 \\
\hline$B H T$ & $T$ & 0.991 & 0.259 & 1.741 & 0.988 & 0.9904 & 0.994 & 0.248 & 1.724 & 0.950 & -- & -- & -- & -- & -- & -- & -- & -- & -- & 2.046 & -- & -- & 0.9964 & 1.64 \\
\hline$T B H Q$ & $T$ & 0.955 & 0.302 & 1.729 & 0.953 & 0.9816 & 0.991 & 0.269 & 1.784 & 0.780 & -- & -- & -- & -- & -- & -- & -- & -- & -- & 1.934 & -0.990 & -- & 0.9953 & 6.01 \\
\hline$E T X$ & $T$ & 0.982 & 0.284 & 1.971 & 0.958 & 0.9820 & 0.991 & 0.628 & 1.241 & 0.525 & -- & -- & -- & -- & -- & -- & 9.187 & -- & -- & -- & -- & -- & 0.9954 & 2.79 \\
\hline TOC & $T$ & 0.993 & 0.271 & 1.715 & 0.997 & 0.9912 & 0.994 & 0.281 & 1.781 & 1.082 & -- & -- & -0.990 & -- & -- & -- & -- & -- & -- & -- & -- & -- & 0.9960 & 1.32 \\
\hline \multicolumn{25}{|c|}{ RESPONSES FOR THE ABTS REACTION } \\
\hline TRO & $C$ & 1.000 & 0.281 & 1.492 & 0.992 & 0.9940 & 0.994 & 0.275 & 1.546 & 0.946 & -- & -- & -- & -- & -- & -- & -- & -- & -- & -- & -0.491 & -- & 0.9953 & 0.44 \\
\hline$A A$ & $C$ & 1.000 & 0.313 & 1.658 & 1.023 & 0.9958 & 1.000 & 0.313 & 1.658 & 1.023 & -- & -- & -- & -- & -- & -- & -- & -- & -- & -- & -- & -- & 0.9958 & 0.00 \\
\hline$P G$ & $C$ & 1.000 & 0.335 & 1.582 & 0.958 & 0.9886 & 0.983 & 0.273 & 1.639 & 0.778 & -- & -- & 4.550 & -- & -- & -- & -- & -- & -- & 2.442 & -- & -- & 0.9935 & -2.11 \\
\hline$B H A$ & $C$ & 1.000 & 0.318 & 1.424 & 1.036 & 0.9939 & 1.000 & 0.318 & 1.424 & 1.036 & -- & -- & -- & -- & -- & -- & -- & -- & -- & -- & -- & -- & 0.9939 & 0.00 \\
\hline$B H T$ & $C$ & 1.000 & 0.286 & 1.376 & 1.034 & 0.9913 & 0.958 & 0.284 & 1.429 & 1.006 & -- & -- & -- & -- & -- & -- & -- & -- & -- & -- & -- & -- & 0.9924 & -2.26 \\
\hline$T B H Q$ & $C$ & 1.000 & 0.206 & 1.070 & 1.010 & 0.9787 & 1.000 & 0.206 & 1.070 & 1.010 & -- & -- & -- & -- & -- & -- & -- & -- & -- & -- & -- & -- & 0.9787 & 0.00 \\
\hline ETX & $C$ & 0.974 & 0.263 & 1.696 & 0.967 & 0.9950 & 0.994 & 0.247 & 1.828 & 0.918 & -- & -- & -- & -- & -- & -0.799 & -0.866 & -- & -- & -- & -- & -- & 0.9958 & 1.47 \\
\hline TOC & $C$ & 0.991 & 0.349 & 2.066 & 1.057 & 0.9976 & 0.989 & 0.680 & 1.311 & 0.957 & -- & -- & -- & -- & -- & -- & 3.519 & -- & -- & -- & 2.498 & -- & 0.9978 & 0.11 \\
\hline TRO & $T$ & 0.995 & 0.268 & 1.684 & 1.017 & 0.9951 & 0.988 & 0.477 & 1.207 & 1.017 & -- & -- & -- & -- & -- & -- & 2.733 & -- & -- & -- & 3.237 & -- & 0.9972 & -0.98 \\
\hline$A A$ & $T$ & 0.983 & 0.289 & 1.874 & 0.986 & 0.9965 & 0.979 & 0.292 & 1.927 & 1.007 & -- & -- & -- & -- & -- & -- & -- & -- & -- & -- & -0.483 & 0.019 & 0.9972 & 1.12 \\
\hline$P G$ & $T$ & 0.993 & 0.331 & 1.861 & 0.995 & 0.9945 & 0.981 & 0.325 & 1.810 & 0.983 & -- & 1.721 & -- & -- & -- & -- & -- & -- & -- & -- & 4.163 & -- & 0.9963 & -1.75 \\
\hline BHA & $T$ & 0.956 & 0.306 & 1.762 & 1.044 & 0.9900 & 0.929 & 0.263 & 1.954 & 1.065 & -- & -- & -- & 0.102 & -- & -- & -0.512 & -- & -- & -- & -- & -- & 0.9955 & 0.18 \\
\hline$B H T$ & $T$ & 0.991 & 0.259 & 1.741 & 0.988 & 0.9904 & 0.994 & 0.248 & 1.724 & 0.950 & -- & -- & -- & -- & -- & -- & -- & -- & -- & 2.046 & -- & -- & 0.9964 & 1.64 \\
\hline$T B H Q$ & $T$ & 0.990 & 0.221 & 1.324 & 1.015 & 0.9945 & 0.965 & 0.298 & 1.091 & 1.192 & -- & -- & -- & 0.038 & -- & -0.537 & -- & -- & -- & -- & -- & -- & 0.9968 & 0.14 \\
\hline ETX & $T$ & 1.000 & 0.270 & 1.899 & 1.012 & 0.9976 & 0.993 & 0.268 & 1.962 & 1.000 & -- & -- & 2.668 & -- & -- & -- & -- & -- & -- & 1.745 & -- & -- & 0.9980 & -0.52 \\
\hline TOC & $T$ & 0.991 & 0.337 & 2.480 & 0.982 & 0.9978 & 0.990 & 0.332 & 2.420 & 0.977 & -- & -- & 1.644 & -- & -- & -- & -- & -- & -- & 1.111 & -- & -- & 0.9979 & -0.09 \\
\hline
\end{tabular}

